
September 1945

\title{
AN APPARATUS FOR PHOTOGRAPHING INTERFERENCE PHENOMENA
}

\author{
By James B. Saunders
}

\begin{abstract}
A photographic instrument is described that was designed for recording the changes produced in the order of interference fringes over long periods and for recording large changes in the order of interference. When this instrument is used with interferometric systems for studying the changes caused in transparent solids by heating, it can be made to yield a continuous record of the simultaneous changes in temperature, time, index, strain, and density. For example, to record those changes that are caused by the annealing of an optical glass at an annealing temperature near the lower part of its annealing range requires several weeks of continuous recording. The total amount of 35-millimeter film needed by the instrument for such a record (covering, say 3 months) does not necessarily exceed 10 feet. To record the expansion of a material that is being heated from room temperature to $500^{\circ} \mathrm{C}$, at a rate of 3 degrees centigrade per minute, requires approximately 1 foot of 35 -millimeter film. Thus, even very long tests require such a small amount of film that the operator is able to process it in any small dark room. The fringes are photographed at their natural size, and the fringe shifts can be determined from the photographic record without the use of enlarging or projection equipment.
\end{abstract}

\section{CONTENTS}

II. Description of the method

III. Description of the method....... 159

III. Description of the apparatus

1. General _.............. 161

2. Optics of the interferograph

(a) Light source

(b) Collimating and focusing lens

(c) Dispersion and deviation prism

(d) Interferometer

(e) Diagonal mirror.

(f) Eyepieces_......... 168

3. Mechanics of the interferograph

(a) Rotation of the constant-deviation prism

(b) Diaphragms and stops for light control........ 168

(c) Camera _...

(d) Focusing mechanism..... 172

4. Furnace

5. Control board and other accessories

(a) Wiring diagram and time switches........... 173

(b) Film-loading key _............. 174

(c) Reading frame

(d) Mounting tripod...

IV. Adjustments of the apparatus 
P. Assembering the

V. Assembling the interferometer

1. Preliminary considerations

2. Assembly procedure

VI. Photographic exposure

1. Selection of photographic film

2. Contrast_.

3. Photographic density of film

VII. Other applications of the interferograph

VIII. Results__.

IX. Proposed refinements

X. Conclusions

XI. References_......... 186

\section{INTRODUCTION}

The increasing application of interferometry to the measurement of physical changes in material has created a demand for an automatic recorder. Visual-observation methods have proved unsatisfactory for extended tests because they demand the continuous and undivided attention of an observer and because of the frequent uncertainty resulting from errors in fringe count. The motion-picture camera, as applied by Nix and MacNair [1] ${ }^{1}$ to the photography of interferometer data, eliminates many of the objections inherent in the visual method. However, this method has certain disadvantages that have prevented its general use. Each individual picture requires an exposure of several seconds, even when the brightest of monochromatic sources of light is used. Consequently, if 20 pictures are required (as was proposed by Nix and MacNair) to record the passing of each fringe, it follows that 1 fringe per 20 times the minimum exposure time is the fastest rate of change in the order of interference that can be recorded. Many tests require a faster means of recording than is possible with a motion picture camera under such conditions. The motion picture method is also impractical for slow changes extending over long periods because the number of pictures per hour cannot be materially reduced without danger of failing to record changes that may occur between exposures. Because of these objections and the cost and difficulties of processing the large amount of film required, this method has not been generally accepted.

Trowbridge [2] proposed a photographic method for recording interference phenomena that permits the recording of much faster changes in the order of interference. Trowbridge's method has the further advantage of continuity over the motion-picture method and requires a relatively small amount of film. Arnulf [17], and later Sinden [18], modified Trowbridge's method but apparently failed to produce a practical instrument. By the addition of several new features, a practical means for recording all types of interference fringes has now been produced.

To fulfill the needs of this laboratory, where the interferometer is used in a large number of tests, the design and construction of an automatic photographic recorder was undertaken. Such an instrument must satisfy certain requirements if it is to be practical. That is, the operation of the apparatus must be reasonably simple; the photographic record (in the form of $35-\mathrm{mm}$ film) must be of such length that the operator can conveniently process it in his own laboratory; the monochromatic light source must be constant, enduring,

1 Figures in brackets indicate the literature references at the end of this paper. 
and sufficiently intense to permit continuous recording of the fringes for long periods; the accuracy of the results must be at least equal to that attainable by visual observation; and the operator must be able, during the recording period, to observe visually and to make necessary adjustments without interfering with the continuity and quality of the record. The instrument described in this paper fulfills all the above requirements and extensive use has proved it to be an exceptionally reliable and easily operated instrument. In the following paragraphs this apparatus will be designated an "interferograph" [3] and the resultant records "interferograms."

\section{DESCRIPTION OF THE METHOD}

If a fringe system, such as is shown in figure $1, A$, is focused on a strip of photographic film, a latent image is formed, which, when properly processed, yields a reproduction of the fringe system. However, if the image is partially intercepted by a screen that has a narrow slit perpendicular to the strip of film and which is placed just inside the focal plane of the fringe system, an exposure will yield a reproduction of only the narrow part of the image, which is transmitted through the slit. Figure $1, B$, shows that part of the image that is intercepted by the screen, whereas figures $1, C$ and $1, D$ show the part transmitted through the slit to the film. If the fringes remain fixed and the film is caused to move perpendicularly to the slit and at a rate that is suitable for proper exposure, the resultant effect is that shown in figure 1, $E$. That is, the part of the fringe system that is transmitted by the slit traces dark and light bands parallel to the direction of motion of the film. On the other hand, if the fringes are caused to move across the plate from bottom to top, in a direction that is parallel to the slit, and the film moves from right to left, the exposed film will yield the reproduction shown in figure $1, F$. That is, the bands, which were traced parallel to the edge of the film while the fringes were static, will make an angle to the edge if the fringes are caused to move. Movements of the fringes in the opposite direction will produce a mirror image of the reproduction shown in figure $1, F$.
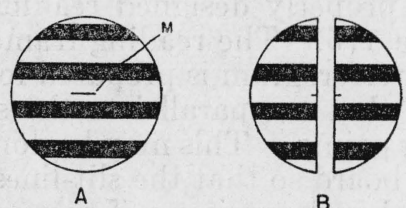

B

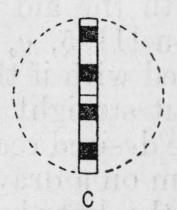

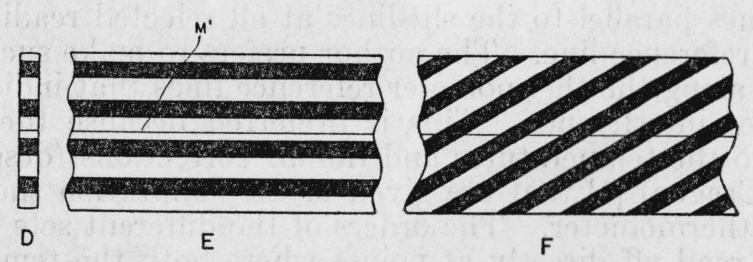

FIGURE 1.-Diagram to show method of producing interferograms from interference fringes.

(See text for explanation.) 


\section{Journal of Research of the National Bureau of Standards}

To determine the fringe movements accurately from the interferograms a reference point is required either in the object plane or in the focal plane of the fringes. Either will serve the same purpose. In most cases a convenient reference point is obtained by scratching a sharp line on the surface of the top interferometer plate, as shown by $M$ in figure 1 , or by placing a fine wire (or some other kind of filament) across the top plate. A cross hair in the focal plane of the camera and very close to the film will serve also. In either case, the image or shadow of this fine line should intersect the slit (approximately $1 / 4 \mathrm{~mm}$ wide) perpendicularly. This intersection serves as a reference point and traces a sharp line, $M^{\prime}$ in the latent image on the film. The line appears black on the positive reproduction and its intersection with a photographically reproduced fringe corresponds to the time when that fringe passed the reference point. If the instrument records more than one phenomenon (in addition to time), such as both temperature changes and expansion, then more than one set of fringes is needed and each set requires an individual reference point. A complete datum from such a record should include the order of each set of fringes at a definite time. In order to locate those points that are photographed simultaneously on the reference lines, transverse lines that are equally spaced and parallel to the slit are produced photographically at definite intervals. Such a line is produced either by cutting off the light source for a few seconds (thus producing a narrow, underexposed strip) or preferably by flashing a small lamp suitably located in the camera box. The latter method is used here. That is, a flash of short duration overexposes a narrow strip equal to the width of the slit. These over(or under-) exposed strips will hereafter be designated "slit-lines." The intersections of the slit-lines with the above-mentioned reference lines serve to locate simultaneously exposed points along the reference lines. The slit-lines are produced automatically and at equal intervals of time by an electrically operated time switch. Since the film moves uniformly, the spacing of the slit-lines affords an accurate measure of time, and simple interpolation is possible for reading points between any two slit-lines.

The data may be read directly from either the negative or a positive reproduction with the aid of a properly designed reading frame, described in section III-5, c, page 175. The reading frame may, however, be dispensed with if the interferogram is prepared for reading by superposing on it straight lines that are parallel to the slit-lines and pass through the desired reading points. This may be done by placing the interferogram on a drawing board so that the slit-lines are parallel to one edge of the board and then by using a T-square to inscribe additional lines parallel to the slit-lines at all selected reading points along either reference line. The author prefers to make such lines at those points along the thermometer reference lines that indicate integral orders of interference. This is preferred because these points correspond to the temperatures and the air corrections (described by Peters and Cragoe) [4] that are given in the calibration sheet of the interference thermometer. The orders of the different sets of fringes can then be read off directly at points where both the temperatures and air corrections are known. 


\section{DESCRIPTION OF THE APPARATUS}

\section{GENERAL}

A diagram of the assembled apparatus is shown in figure 2 and a photograph of it is presented in figure 15. The several parts are shown in greater detail in additional figures and in all of these drawings a

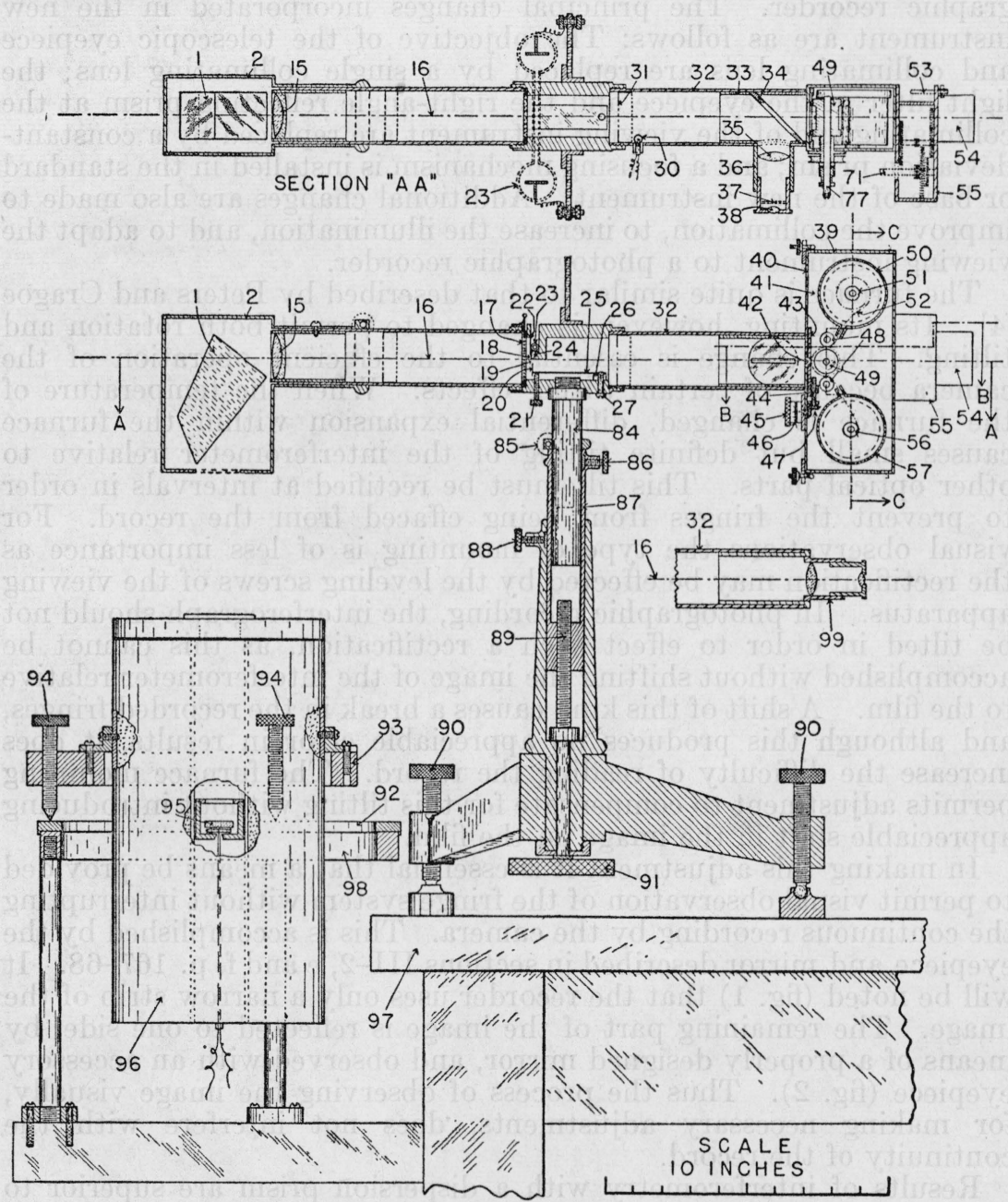

FIGURE 2.-Interferograph and furnace.

1, Constant-deviation prism; 2, prism housing; 15, collimating lens; 16 , optic axis; 17 , iris diaphragm; 18 and 22 are, respectively, the effective source and its semiadjustable jaw; 19 , adjustable slit for the isolation of spectral lines; 21 , knurled screw for moving adjustable jaw $20 ; 23$, spectrum tube; 24 , right-angle reflecting prism; 26 and 27 are, respectively, a block and screw for holding the phosphor bronze strip 25; 30, flash lamp; 31 , diaphragm; 32 and 33, telescoping tubes; 34, diagonal mirror; 35,37 , and 38 are, respectively, the collecting lens, shutter, and eye-lens of eyepiece 36; 43, semiadjustable jaw of exposing slit 42; 44, 45, 46, and 47 are, respectively, the screw, knurled head, scale, and right-angle gears for controlling the width of exposing slit $42 ; 48$, two idling rollers; 49 , sprocket; 50 , cartridge which houses the supply spool $51 ; 52$, photographic film or paper; 53, electrical contact post; 54 , motor; 55 , gear housing; 57 , cartridge which houses the take-up spool 56; 71, gear shaft; 77, sprocket shaft; 84 and 87 , telescoping shaft and tube, respectively; 85,86 , and 88 , clamping screws; 89 , focusing screw with knurled head $91 ; 90$ and 94 , leveling screws of the instrument and furnace, respectively; 92, rotatable ring; 93 and 98, supporting rings; 95 , interferometer; 96 , furnace; 97 , table; 99 , removable eyepiece. Sections $B B$ and $C C$ are shown in figures 9 and 8 , respectively. 
reference number always indicates the same part of the apparatus. In making thermal expansion measurements the complete apparatus, for procuring the interferograms, consists of the interferograph, an electric furnace, an electric control board, and several accessories.

The interferograph is essentially a modification of the viewing instrument, described by Merritt [5], with the addition of a photographic recorder. The principal changes incorporated in the new instrument are as follows: The objective of the telescopic eyepiece and collimating lens are replaced by a single collimating lens; the light filter in the eyepiece and the right-angle reflecting prism at the collimating end of the viewing instrument are replaced by a constantdeviation prism; and a focusing mechanism is installed in the standard or base of the new instrument. Additional changes are also made to improve the collimation, to increase the illumination, and to adapt the viewing instrument to a photographic recorder.

The furnace is quite similar to that described by Peters and Cragoe [4]. Its mounting, however, is changed to permit both rotation and tilting. This change is essential to the efficient operation of the camera because of certain tilting effects. When the temperature of the furnace is changed, differential expansion within the furnace causes small but definite tilting of the interferometer relative to other optical parts. This tilt must be rectified at intervals in order to prevent the fringes from being effaced from the record. For visual observations the type of mounting is of less importance as the rectification may be effected by the leveling screws of the viewing apparatus. In photographic recording, the interferograph should not be tilted in order to effect such a rectification, as this cannot be accomplished without shifting the image of the interferometer relative to the film. A shift of this kind causes a break in the recorded fringes, and although this produces no appreciable error in results, it does increase the difficulty of reading the record. The furnace mounting permits adjustment to compensate for this tilting without introducing appreciable shift in the image on the film.

In making this adjustment it is essential that a means be provided to permit visual observation of the fringe system without interrupting the continuous recording by the camera. This is accomplished by the eyepiece and mirror described in sections III-2, e and f, p. 167-68. It will be noted (fig. 1) that the recorder uses only a narrow strip of the image. The remaining part of the image is reflected to one side, by means of a properly designed mirror, and observed with an accessory eyepiece (fig. 2). Thus the process of observing the image visually, for making necessary adjustments, does not interfere with the continuity of the record.

Results of interferometry with a dispersion prism are superior to those obtainable with a light filter. For interferometer tests in which the observations are made visually, it is generally sufficient to use a good source of monochromatic light and a suitable light filter. For photographic recording, however, the distribution of the spectral sensitivity of the film makes necessary a higher degree of homogeneity in the light, and it is desirable, therefore, to use a dispersion prism. To obtain a large dispersion, a constant deviation prism of the Pellin and Broca [6] type is used to replace the right-angle prism of the ordinary instrument. The light passes through the dispersion prism twice, thus producing sufficient dispersion to isolate any 
spectral Tine in the source if its nearest neighbor differs in wavelength by as much as 50 angstroms. The selection of any line is accomplished by a simple rotation of the prism about an axis which is so chosen that image shifts are negligible.

Visual observations at high temperatures are more difficult with filtered light (used by most of the present-day viewing instruments) than with dispersed light. A filter that will eliminate the incandescent glow of a hot furnace and also isolate a spectral line will usually absorb a large percentage of the light from the transmitted band, thus making both visual and photographic observation difficult. That is, any filter that transmits only a narrow band will also absorb a large portion of the light in this band. If a dispersion prism is used for monochromatization, a filter that absorbs practically all light of long wavelength and that transmits a large percentage of the light of short wavelength may be used to eliminate the incandescent light of the furnace without serious absorption of a selected line of short wavelength. However, a filter is not necessary for photographic work unless panchromatic film is used. By using noncolor-sensitized film (insensitive to the greens, yellows, and reds) with a blue line of the source, only light from this blue line has any effect on the film unless the furnace temperature exceeds $1,100^{\circ} \mathrm{C}$. Consequently the result is a clear photograph, free from haze or fog. A filter may still be needed in the eyepiece for visual adjustments.

\section{OPTICS OF THE INTERFEROGRAPH}

The essential optical parts of the interferograph are shown in figure 3. Light from the source, 23, after being reflected by the rightangle reflecting prism, 24 , and collimated by the lens, 15 , passes through the constant-deviation prism, 1 , to the interferometer, 95 . The normal to the center of the interferometer is assumed to form a

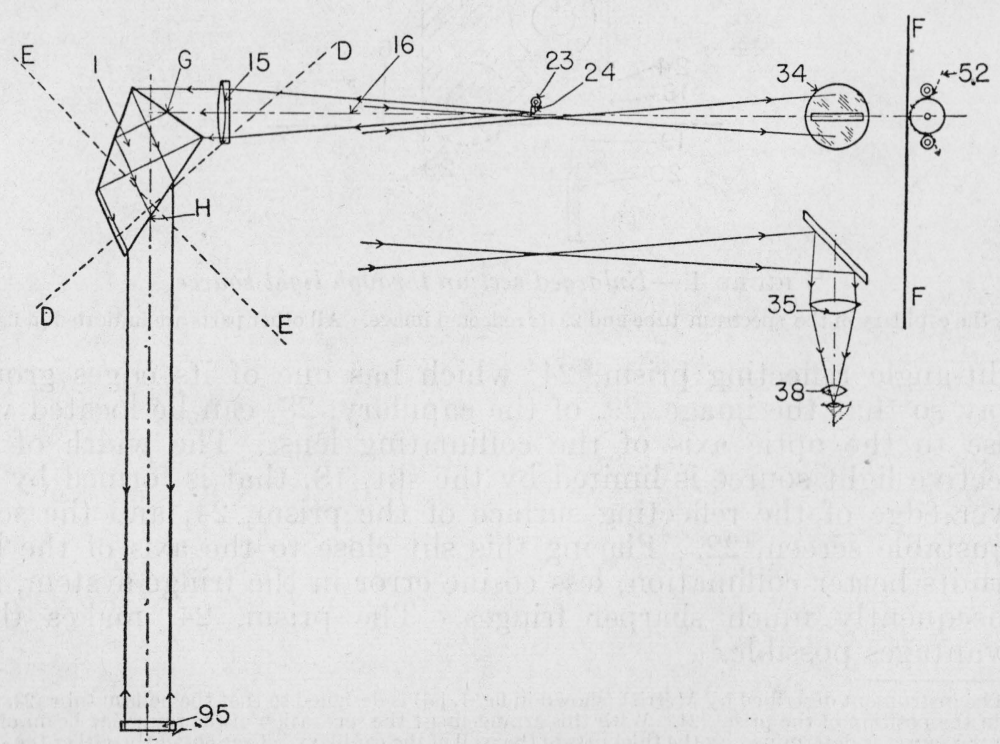

Figure 3.-Optical parts oj the interferograph.

$F F$, image plane; $G$ and $H$, entrance and exit points, respectively, of prism $1 ; E E$ and $D D$, the loci along which the axis of rotation of prism 1 may be chosen for climinating, respectively, axial and longitudinal shifts of the image. All other parts are indicated in figure 2. 


\section{Journal of Research of the National Bureau of Standards}

perpendicular intersection with the optic axis of the collimating lens. The light is dispersed on passing through prism 1. By rotating this prism about an axis perpendicular to the plane of this figure, any one of the refracted beams, corresponding to the different spectral lines of the source, may be caused to fall perpendicularly onto the interferometer plates. On reflection from these plates this beam will retrace its path through the prism to the source. The light beam from neighboring lines will also return through the prism with increased dispersion. From each of these beams, lens 15 will form an image of the source in a vertical plane near the source. By rotating prism 1, any one of these images may be caused to fall on slit 19 (fig. 2), and the light from this line will then pass on to the diagonal mirror, 34. A slit in this mirror transmits a narrow rectangular part of this beam to the film, 52 . The remaining part is reflected by the mirror to the collecting lens, 35, which converges the light to a cross section that is sufficiently small for simultaneous observation of all rays from the entire field. The eye lens, 38 , enables visual observation of the interference phenomena.

\section{(a) LIGHT SOURCE}

The light source ordinarily used with this apparatus is a Pleucker tube of helium gas. This tube (23, fig. 4) rests on a long, slender,

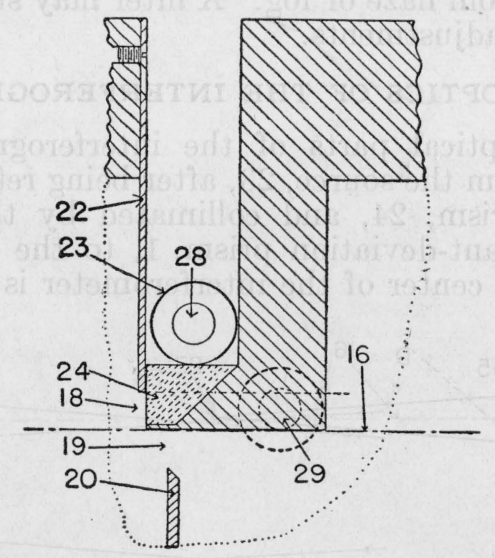

FIgURE 4.-Enlarged section through light source.

28 is the capillary of the spectrum tube and 29 its reflected image. All other parts are indicated in figure 2

right-angle reflecting prism, $\mathbb{2 4}$, which has one of its edges ground away so that the image, 29 , of the capillary, 28 , can be located very close to the optic axis of the collimating lens. The width of the effective light source is limited by the slit, 18, that is formed by the lower edge of the reflecting surface of the prism, 24, and the semiadjustable screen, 22. Placing this slit close to the axis of the lens permits better collimation, less cosine error in the fringe system, and consequently much sharper fringes. The prism, 24, makes these advantages possible. ${ }^{2}$

2 The instrument described by Merritt (shown in fig. 1, [5]) is designed so that the helium tube (23, fig. 4) lies in the position of the prism, 24. With this arrangement the separation of the returning beam of light from the source is determined by the thickness of the wall of the capillary. Consequently, either the source or its image (produced by light returning to the focal plane of the collimating lens) must be some distance from the optic axis; thus, a larger cosine error is produced in the collimated light. By introducing a slender prism similar to prism 24, it was found that the maximum usable separation of interferometer plates (length of specimen) was doubled. A corresponding improvement in fringe definition was also attained for any given separation of plates. 
The effective shape of the light source commonly used for interference measurements with reflected light is that of a half-moon [7]. This shape is considered best for securing maximum intensity with a given cosine error and is entirely satisfactory if the spectral line employed has no close neighbors. However, it is sometimes necessary to use a narrow slit-like source for the isolation of close spectral lines. This can be done without sacrificing intensity if all but a narrow slit along the capillary of the spectral tube is silvered.

In fact, a considerable increase in the directed light from a spectral tube is produced by silvering the back of the capillary. If one-half (180 degrees of arc) of the capillary is silvered, the intensity of the light that is effective in producing the interference fringes is approximately doubled. If two-thirds (or 240 degrees of arc) of the capillary is silvered, the intensity is approximately trebled. Up to a certain point, further narrowing of the unsilvered aperture of the capillary produces a still further increase in the density of the source. ${ }^{3}$ This increase in surface density is limited, however, by the increasing absorption resulting from the increasing number of internal reflections before the light emerges from the narrowing slitlike window. Thus, the silvered capillary permits the use of a narrower source and a greater intensity for a given cosine error. A decrease in width of the source and an increase in surface density assist in the isolation and use of close and less intense lines from a multiline source.

In preparing a narrow slit of this kind, the capillary is silvered over its whole circumference for a length of approximately $1 \mathrm{~cm}$ near the middle. The silver is then coated with shellac, or any other suitable coating, for protection. After the coating is dry, an axial slit with straight edges and with the desired width is then produced by removing a narrow strip of the silver with a sharp razor blade. In the case of the well-separated helium lines, a width of $2 \mathrm{~mm}$ is suitable.

\section{(b) COLLIMATING AND FOCUSING LENS}

The collimating lens $(15$, fig. 3$)$ is a cemented doublet, corrected for chromatic and spherical aberration. Light reflected from its surfaces ${ }^{4}$ forms ghost images, but these do not interfere with the photographic record if they are made to fall below the exposing slit. The placement of the optical parts is arranged so that the collimating lens also serves as the focusing lens. Fortunately, this arrangement is convenient both for obtaining the desired magnification and for properly locating the controlling aperture (18, fig. 4). The number of optical parts is thus reduced to a minimum.

(c) DISPERSION AND DEVIATION PRISM

If a right-angle reflecting prism ( $R$, fig. $5, A)$ is combined with the two parts, $P 1$ and $P 2$, of a 60 -degree dispersion prism (fig. $5, B$ ) in the manner shown by figure $5, C$, the result is a prism having both the dispersion properties of the dispersion prism in figure $5, B$, and the right-angle deviation properties of the reflecting prism, $R$. The path of the ray of light shown in the reflecting prism is that for which the prism produces no dispersion. The path of the ray of light shown

\footnotetext{
${ }^{3}$ This method of increasing the effective illumination has also been applied to mercury vapor lamps. It is recommended for all such sources where increased illumination from spectral lines is desired.

It is desirable to give this lens the treatment described by Cartwright [8] for extinguishing reflection of light from glass.
} 
in the dispersion prism is that for which the prism produces minimum deviation. For the combination, shown in figure $5, C$, each part of the dispersion prism produces both the same dispersion and deviation as shown in figure $5, B$, but as the beam is inverted by reflection before entering the second half of the dispersion prism, the deviation of this half cancels the deviation of the other, whereas the dispersions are still additive, as in figure $5, B$. The dispersion prism in figure $5, B$, may be set for minimum deviation of a ray of any other wavelength by a simple rotation, and when thus rotated the new ray passes along the same path within the prism as that previously followed by the first-mentioned ray. The same is true for a rotation of the constantdeviation prism, except that no change is produced in the total deviation. The prism used with this interferograph, and shown in figure $5, D$, has the same properties as that shown in figure $5, C$, and is a single piece of glass.

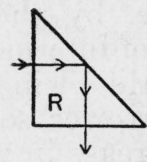

A

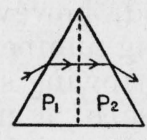

B
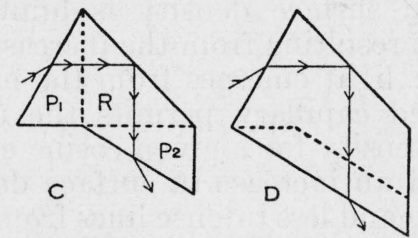

Figure 5.-Properties of the constant-deviation prism.

$A$, right-angle reflecting prism; $B, 60$-degree dispersion prism; $C$, constant-deviation prism compounded from parts of $A$ and $B ; D$, constant-deviation prism made from a single piece of glass.

A rotation of the constant-deviation prism (1, fig. 3) in changing from one line to another generally produces an axial shift in the image plane of the interferometer. This is caused by a change in the effective object distance. That is, in general, both the air and glass paths, comprising this distance, change with the rotation of the prism. If, however, the axis of rotation is chosen anywhere along the line $D D$, the change in the air path cancels the change of path in glass to a close approximation. Rotation of this prism also produces a lateral (vertical in this case) displacement of the interferometer image. This displacement is caused by a lateral shift in the rays on emerging from prism 1. For example, when the prism is in the position shown, the axial ray of the collimating lens emerges as the axial ray of the interferometer plate and the image points of these coincide in the image plane, $F F$. If the prism is now rotated clockwise about the entrance point, $G$, of the axial ray, this ray, now of another color, but deviated by 90 degrees, still follows the path traced through the prism to the exit point, $H$, and emerges parallel to the axis of the interferometer plate; but the point $H$ has been moved to the left by this rotation. Thus, the axial ray of the collimating lens and that of the interferometer plate will be imaged at two different points, one above the other, in the image plane, $F F$. In general, rotation about any other point also causes a shift in the exit point of the axial ray, 16. It has been found, however, that if the axis of rotation is chosen along the line $E E$, the lateral shift of the image caused by the rotation of the prism may be minimized to the same degree as the axial shift was minimized by the means described above. Fortunately, the intersection of these two lines, $E E$ and $D D$, is so located relative to the 
prism that it can be chosen as the position of the axis of rotation for the prism. As the result of this choice, both shifts are practically eliminated.

The purpose of eliminating any image shift is to improve the application of the interferograph for measuring the length of samples to a very high degree of accuracy by means of the "theory of coincidence," described by Perot and Fabrey [9]. However, the lateral shift of the image does not affect ordinary visual observations.

\section{(d) INTERFEROMETER}

The recording of time is automatic with the interferograph, as described in section II, page 160 . Consequently, if the data to be recorded consist only of temporal changes in any single quantity that may be observed with an interferometer, then one set of fringes is sufficient. However, if two or more kinds of such changes are to be recorded, additional sets of fringes are necessary.

Two sets of fringes are required for recording both thermal expansion and temperature by interferomeiry. In figure 6 the lower plate, $T$, serves as an interference thermometer [10], and the expansion fringes, showing the expansion of specimen $S$, are produced in the usual manner by light reflected from the top surface of $T$ and the bottom

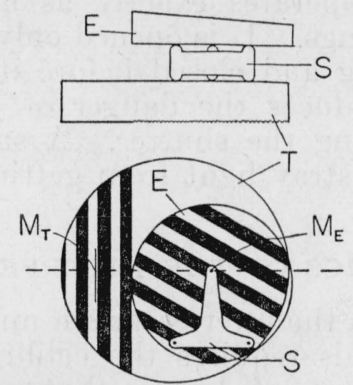

FIGURE 6.-The interferometer.

$E$, An interferometer plate; $S$, test sample; $T$, interference thermometer; $M_{T}$ and $M_{E}$, reference lines of the temperature and expansion fringes, respectively.

surface of plate $E$. The broken line in figure 6 represents the position of the exposing slit relative to the two reference points, $M_{T}$ and $M_{E}$, of the temperature and expansion fringes, respectively. The thermometer reference point, $M_{T}$, is the intersection of the reference mark ( $M$, fig. 1, $A$ ) with the indicated position of the exposing slit. The reference mark should be sufficiently long so that an appreciable rotation of the interferometer about $M_{E}$ may be effected without removing the image of $M_{T}$ from the exposing slit. Also the reference mark should be parallel to the fringes so that the rotation causes no change in the order of the thermometer fringes relative to their reference point.

\section{(e) DIAGONAL MIRROR}

The diagonal mirror (34, fig. 2), used for intercepting a part of the light of the interferometer for visual adjustments or observation, may be made either of silvered glass or stainless steel. The slit, through its center, should be wide enough to permit some latitude in the adjustment of the exposing slit and also for visual adjustment of the 
image in the slit before closing the camera to begin a recording. A $1.5-\mathrm{mm}$ slit in the mirror has been used successfully.

\section{(f) EYEPIECES}

Two eyepieces ${ }^{5}$ (36 and 99, fig. 2) of identical optical design, and corrected for spherical aberration, are used with the interferograph. Each consists of a collecting lens and an eye lens that are separated by their common focal lengths of $5 \mathrm{~cm}$. The collecting lenses are sufficiently large to cover the whole image, whereas the eye lenses are quite small.

Eyepiece 99 is designed for easy removal and, after the interferometer is assembled, it is replaced by the photographic recorder and diagonal mirror. When this eyepiece is in its position for visual work, its collecting lens is in the image plane of the interferometer. Eyepiece 36 is permanently attached to the side of the interferograph, as shown in figure 2. It is used, during the recording period, for observing the interferometer and for making adjustments when necessary. The collecting lens of this eyepiece is located in the reflected image plane of the interferometer. This eyepiece has a shutter, located between its lenses, that is controlled by a mechanism similar to that used in cameras having the between-the-lens type of shutter. The shutter operates exactly as a camera shutter with "bulb" and "time" settings. It is opened only after the eye is placed in position for observing and closed before its removal. A camera release cable greatly reduces the danger of jolting the instrument when opening and closing the shutter. A small exit pupil and an eyecup help to prevent stray light from getting to the photographic film while observing.

\section{MECHANICS OF THE INTERFEROGRAPH}

For efficient operation the interferograph must permit close control of light conditions. This requires the calibration of several scales which indicate the settings of devices that control the amount and quality of the light producing the record. Also it is important that these controls be operative without affecting the continuity of the record.

(a) ROTATION OF THE CONSTANT-DEVIATION PRISM

The constant-deviation prism (1, fig. 7 ) is fastened between two plates with two layers of felt to serve as padding. Two pressure springs, 6 and 12, tend to rotate the prism clockwise about its bearing shaft, 10. The rotary motion is limited by the lever arm, 8 , which bears against pin 7 on plate 3 and is rotatable about screw 5 . The rotation of this lever arm is controlled by the motion of the micrometer screw, 13. Pressure springs, 6 and 12, maintain permanent contact between the arm, 8 , and both the micrometer screw, 13 , and pin, 7. Thus, all back lash is eliminated and the accurate calibration of the micrometer head, 14, against the wavelength of known spectral lines is possible. The bearing shaft, 10 , is held in place by a ring, 9 , and set screw, 11.

\section{(b) DIAPHRAGMS AND STOPS FOR LIGHT CONTROL}

Figure 4 shows a cross section of the spectrum tube, 23 , with the unsilvered slit facing downward. The reflected image of this slit

\footnotetext{
By modifying the mounting of eyepiece 36 it could serve both purposes, thus eliminating eyepiece 99 .
} 
faces the collimating lens which is to the left. Slit 18 is slightly narrower than this reflected slit and is, therefore, the effective source. The area of this slit-like source of fixed width is controlled by means of an iris diaphragm (17, fig. 2). It is important that the size of the iris diaphragm be readily controlled. This is accomplished by means of a lever in combination with a calibrated scale.

The light from the effective source (18, fig. 2) is collimated by lens 15 and dispersed and deviated by prism 1 . It then travels vertically to the interferometer where it is reflected back through prism 1 which further increases the dispersion and causes another right-angle deviation. Lens 15 then produces, in a vertical plane through 18, inverted images of the light source, one corresponding to each spectral line in the source. A rotation of prism 1 moves these images vertically. By observing through eyepiece 99 , the operator may cause any one of these images to coincide with aperture 19.

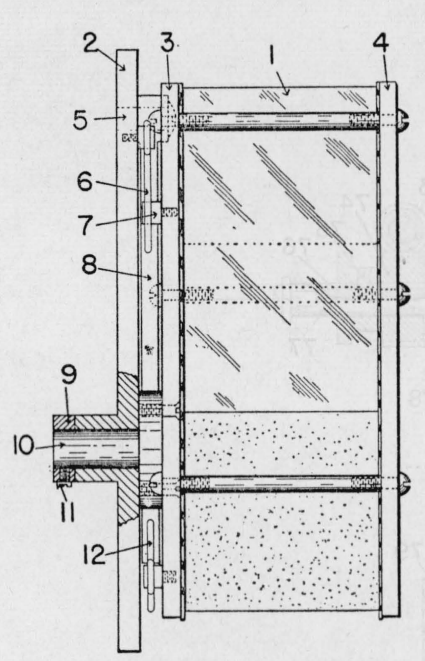

A

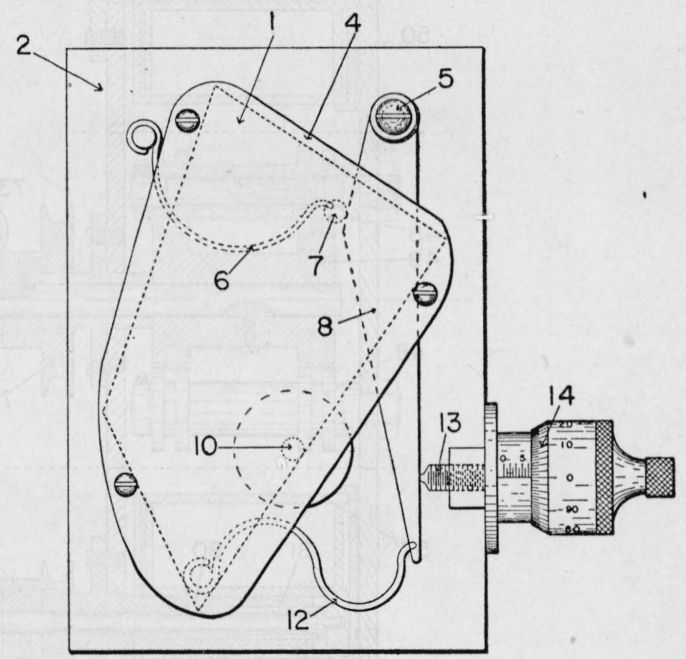

B

FIGURe 7.-Mounting of the constant-deviation prism.

3 and 4, Plates for holding the prism in place; 5 , pivot screw; 6 and 12, springs exerting thrusts on pin 7 and lever arm 8, respectively; 9 and 11, ring and set screw, respectively, for preventing axial motion of shaft 10 , on which the prism rotates. The rotation of the prism is controlled by means of screw 13 with the aid of a micrometer scale 14.

The width of slit 19 is determined by the position of screen 20 which is controlled by means of knurled headed screw 21 (fig. 2). This screen is attached to a phosphor bronze strip, 25, which maintains contact with screw 21 and is securely fastened to the instrument through block 26 and set screw 27 . The length of slit 19, which in operation is approximately equal in width to that of 18 , is also controlled by iris diaphragm 17 .

Additional diaphragms are placed in suitable parts of the instrument to prevent stray light from reaching the photographic film. One such diaphragm is shown by 31 in figure 2 . All inside parts of the instrument are painted a dull black. 
(c) CAMERA

The front part, 40 , of the camera box is brazed to tube 33 , in which the diagonal mirror is located. Tube 33 telescopes into tube 32 and is replaceable by eyepiece 99 for visual work. Both the camera box and prism housing 2 may be rotated 90 degrees about the optic axis, 16 , of the interferograph. This permits the instrument's use for recording with vertical-type interferometers.

The essential elements of the camera box are shown in figures 2 and 8. It is lighttight and made in two sections which may be

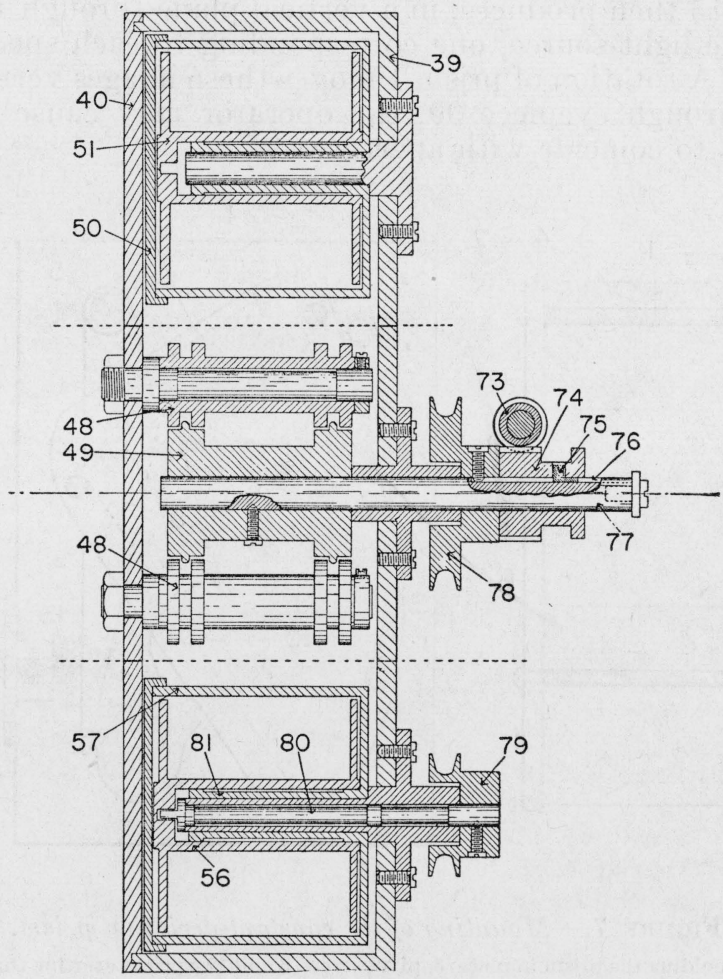

Figure 8.-Camera box (section CC of fig. 2)

73, Worm; 74, worm gear; 75, key; 76, keyway; 39 and 40, the two separable sections of the camera box; 78 and 79 , slip-belt pulleys. All othêr parts are indicated in figure 2.

separated. In viewing the camera box in the direction indicated for observing section $C C$ of figure 2, the top, bottom, back, and righthand sides form one of these sections, 39, which is referred to as the removable part, and the front and left-hand sides form the other section, 40, which is referred to as the fixed part as it is brazed to tube 33 .

The film spools are housed in lighttight cartridges, 50 and 57, which can be removed from the camera box without exposing the film. The slots through which the film is fed into and out of the cartridges are lined with a deep, black plush to prevent light leakage. The capacity of each spool is approximately 35 feet of photographic film. An 
extra take-up spool with its cartridge permits the processing of one record while making another.

The mechanism for moving the film is shown in figure 9. The power for driving sprocket 49 , which imparts uniform motion to the film, is supplied by a small synchronous motor, 54 , which by means of gear 58 drives gear 59 , the shaft of which also carries gears 60 and 61 . The three gears, 62,63 , and 64 are fastened rigidly to a sleeve, 65 , that can be moved along a shaft, 71 . This movement permits the alternative engagement of three pairs of gears, 59-62, 60-63, and 61-64, each of which gives a different gear ratio and consequently permits the selection of three different speeds for the movement of the photographic film. Sleeve 65 is constrained to turn with shaft 71 by a set screw, 67 .

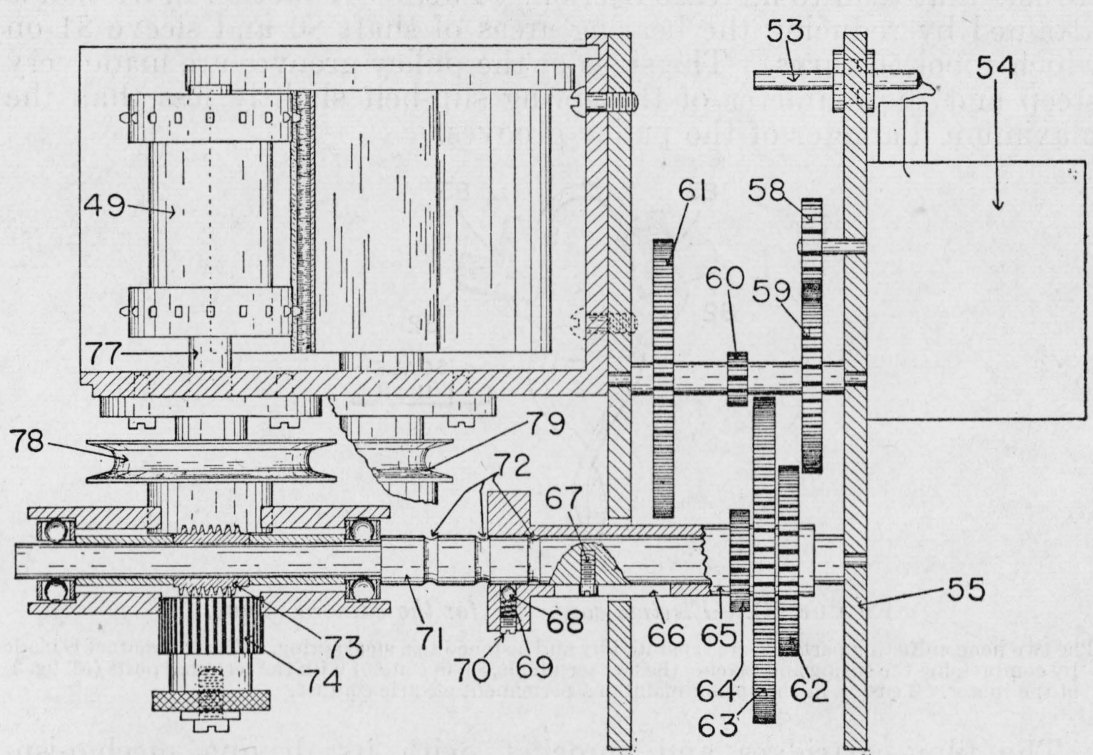

FIGURE 9.-Film-driving mechanism (section BB of fig. 2).

58 , Transmission gear that drives the set of gears 59,60 , and 61 at a fixed speed; 62,63 , and 64 , a second set of gears that rotate together with sleeve 65 and can be made to engage gears 59,60 , and 61 , respectively, by moving sleeve 65 along shaft $71 ; 66$, groove; 67 , pin; 68 , steel ball that is pressed against shaft 71 by pressure spring 69 and set screw 70 . All other parts are indicated in figures 2 and 8.

Slot 66 , which is parallel to the axis of the sleeve in which the head of set screw 67 is located, permits movement of the sleeve along the shaft without relative rotation. A steel ball, 68, and its spring, 69, holds the sleeve in position when the gears are meshed as its position then coincides with one of the three grooves, 72 . The sprocket, 49 , and worm gear rotate with shaft 77 .

In order to bring unexposed film into position before starting a record and also to wind up that part of the exposed film that is between the exposing slit and the take-up cartridge at the end of a recording, it is necessary to move the film manually, independent of the driving mechanism. To accomplish this the worm gear can be disengaged from the worm in the manner shown in figure 8. That is, the worm gear can slide along shaft 77 while key 75 , which fits into the keyway, 76, constrains the two to rotate together in either its $663099-45-2$ 
engaged or disengaged positions. Thus, the sprocket may be rotated manually when the worm gear is disengaged. In order to move the film a small known amount, ball 68 may be set half way between any two of the adjacent grooves 72 (as in the position shown in fig. 9 ), thus disengaging the gears. With the worm gear engaged, sleeve 65 may be rotated manually, causing the film to move. A 360-degree rotation of the sleeve will then move the film exactly one-tenth of an inch.

The take-up spool (56, fig. 8) is powered through the V-shaped pulleys, 78 and 79 , which are connected by a spring slip-belt (not shown). These pulleys are constructed so that the plane of the belt falls within the bearing areas of their shafts. This eliminates side thrusts that tend to increase friction. Further reduction in friction is attained by reducing the bearing areas of shaft 80 and sleeve 81 on which spool 56 turns. The sides of the pulley grooves are made very steep and the diameter of the spring slip-belt slightly less than the maximum diameter of the pulley grooves.

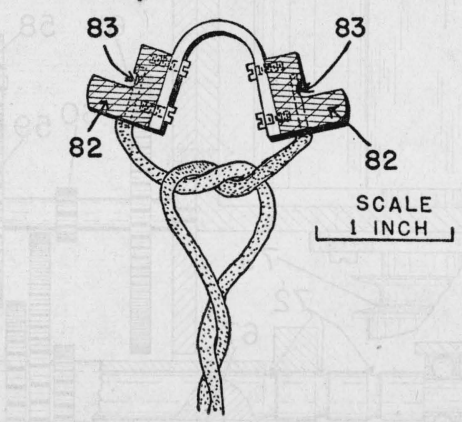

FIGURE 10.-Electric connector for the camera motor.

The two nonconducting parts, 82 , are separated by and fastened to a steel spring. Electric contact is made by compressing the spring and placing the two terminals, 83 , in contact with the terminal posts (53, fig. 2 ) of the motor. Tension in the spring maintains permanent electric contact.

The film cartridges and sprocket, with its driving mechanism (motor and transmission gears), are attached to the removable part of the camera box (fig. 2), and the two floating rollers and exposing slit with its controls are attached to the fixed part. This arrangement permits both easy access to the slit for its adjustment relative to the image of the interferometer and easy threading of the film between the rollers, 48 , and the sprocket wheel.

When loading and unloading the camera, it is desirable to disconnect the electric power. Also it is important to guard against moving the instrument while remaking the connection. The terminals of the motor are connected to two parallel posts (53, fig. 2). The attachment, shown in figure 10, for electrifying these parts is light and allows connections to be made without danger of shaking the instrument. The other end of the electric cord is plugged directly into the 110-volt alternating-current power line.

\section{(d) FOCUSING MECHANISM}

Focusing of the interferograph (fig. 2) is accomplished by varying the object distance, which is the optical distance between the interferometer, 95, and the collimating lens, 15. This may be done 
either by raising or lowering furnace 96 by means of the three leveling screws, 94 , by raising or lowering the interferograph by means of its three leveling screws, 90 , by sliding shaft 84 along inside of tube 87 , or by operating the elevating screw, 89 , by means of wheel 91 . Only the latter two means are used in practice, one for coarse adjustment and the other for fine adjustment.

\section{FURNACE}

Figure 2 shows the furnace, partly in elevation and partly in section. It is supported by a ring, 93, which in turn is supported by three equally spaced leveling screws. These screws rest on ring 92 which fits on and over another ring, 98. Ring 92 is rotatable about ring 98. An overhanging flange constrains the axis of the two to coincide at all times. The rotatable top ring carries the furnace and the bottom ring is fastened rigidly to supporting parts which are firmly attached to the table on which the interferograph is supported. The electric connections (thermocouple and heater leads) to the furnace are made flexible so as to prevent breakage from flexure caused by rotating the furnace. The interferometer, 95, is near the center of the furnace and on a level with the rotatable ring, 92.

\section{CONTROL BOARD AND OTHER ACCESSORIES}

(a) WIRING DIAGRAM AND TIME SWITCHES

A wiring diagram of the control board is shown in figure 11. The Variac transformer, $T_{1}$, is well suited for automatic control of heating rates. The intensity of discharge in the spectrum tube, $H$, is controlled by means of a slide-wire rheostat, $R_{2}$, in series with the primary of a 12,000-volt neon-sign transformer. A control of the intensity of the source is necessary because such control is the best means of adjusting the exposure to the several rates of film speeds. The timeswitch transformer, $T_{3}$, delivers 6 volts across its secondary. This voltage is divided between the time-lamp, $F L$, and the resistance, $R_{3}$ (a 100 -ohm, radio-type, slide-wire rheostat) so that the lamp may be made relatively faint when fast films are used.

The slit lines, defined in section II, page 160, are produced by causing the time-lamp, 30 , ( $A A$, fig. 2$)$ to flash on momentarily. Two switches make it possible to control this lamp either automatically or manually. The manually operated switch is used to record the time of incidental events such as a change in heating rate and the automatic switch records the passage of time intervals only. The automatic time switch was constructed from an electric clock. The contact point $\left(C_{1}\right.$, fig. 11$)$ makes contact with the second hand, $S H$, once each minute. The duration of this contact is approximately 1 second. The contact, $C_{2}$, makes contact with the modified minute hand, $M H$, once in each 30 minutes. The duration of this contact is approximately 1 minute. The $C_{2}$ contact is made when the second hand is approximately 180 degrees from its contact position; consequently, the $\mathrm{C}_{1}$ contact is made near the middle of the period of contact at $C_{2}$; thus the possible danger of two $C_{1}$ contacts during one of the $C_{2}$-contact periods is eliminated. With this adjustment made, the time-light is on for 1 second at accurate intervals of 30 minutes. If more or less frequent intervals are desired, the number (two in the case described) of contacts on the modified minute hand must be 
changed accordingly. A disk, having any number of such contact points, would produce that number of flashes during each hour. The manually operated time switch is of the push button or doorbell type.

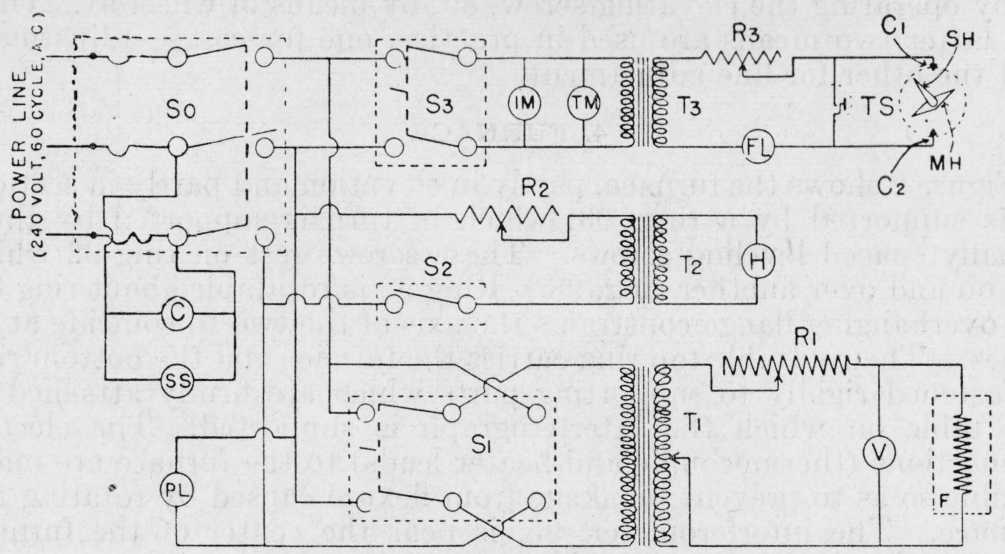

FIGURE 11.-Wiring diagram of the control board.

$S_{0}$, Triple pole, single throw switch; $S_{1}$, double pole, double throw switch; $S_{2}$, single pole, single throw switch; $S_{3}$, double pole, single throw switch; $T_{1}$, variable transformer, Variac; $T_{2}$, neon sign transformer; $T_{3}$, door bell transformer; $R_{1}, R_{2}$, and $R_{3}$, variable rheostats; $C$, electric clock; SS, spare socket; $P L$, panel board light; $I M$, interferograph motor; $T M$, time-switch motor; $F L$, flash lamp; $T S$, time switches: automatic and manual; $M H$, minute hand; $S H$, second hand; $H$, spectrum tube (helium); $F$, electric furnace; $V$, voltmeter.

\section{(b) FILM-LOADING KEY}

A film-loading key (fig. 12) is convenient for loading the supply cartridge with unexposed film. This key is shaped so that it engages the spool in the same manner as shaft 80 of figure 8 . The film is attached to a hook on the spool, which is then inserted into the cartridge, with the film passing out through the plush-lined slot. The

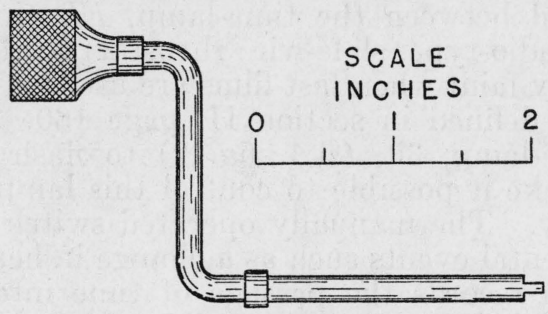

FIGURE 12.-Film-loading key.

cartridge is then closed and the film wound onto the supply spool until full.

Notations are made on the emulsion side of the film, after each ten turns, by means of a stylus. ${ }^{6}$ This serves to keep the operator informed regarding the amount of film remaining after each successive record is cut off and developed. Similar notations, that identify the individual tests, are written on the protruding end of the film at the end of each record. One loading of the supply cartridge serves for

6 This stylus may be similar to that used with an autographic camera and the notations develop in the same way as the autographs do from such cameras. A hard lead pencil also serves excellently in making these notations. 
many complete records as each of these usually requires only 1 to 2 feet of film.

\section{(c) READING FRAME}

In section II, page 160 , one method is described for transforming the interferograms into numerical data. The record may also be read directly from the negative with the aid of a reading frame. This consists of two plates of glass that are separated approximately one-half millimeter by two strips of cellulose (or any other suitable material). The separator strips have their adjacent edges parallel, straight, and separated by a distance slightly greater than the width (35 mm) of the photographic film used. The film is moved through the rectangular slot so produced. A straight mark on one of the glass plates, parallel to the slit lines, serves in determining the fringe orders of the several sets of fringes corresponding to any desired intermediate time or any desired order of a set chosen for any particular reason. The usual procedure, in thermal expansion work, is to read the order (whole number and fraction) of the expansion fringes corresponding to chosen integral orders of the interference thermometer fringes.

\section{(d) MOUNTING TRIPOD}

A tripod used in mounting and arranging the interferometer is an important adjunct. It consists merely of a flat-topped tripod with adjustable feet. Its height is adjusted so that the object distance will remain unchanged when the interferometer is transferred to the furnace. This tripod is placed near the furnace so that the constantdeviation prism may be swung around to a position directly over it merely by rotating the interferograph on its base about shaft 84 . The interferometer system is assembled on this tripod and adjusted while being observed through eyepiece 99 .

\section{ADJUSTMENTS OF THE APPARATUS}

When the interferograph is used for expansion work only, some of the necessary adjustments are final, whereas others must be repeated for each new sample or test. The interferograph may be leveled by the use of two horizontal mirrors, formed by shallow dishes of clean mercury. One mirror is placed on the tripod and the other replaces the interferometer in the furnace. After the instrument is once levelled the levelling screws, 90, should remain unmolested. By replacing the mercury mirror, on the tripod, with an optical flat and observing the reflected light, from this mirror, the instrument is collimated by adjusting the position of lens 15 until the field, observed in the eyepiece, is uniformly illuminated. The instrument is focused by adjusting the height of the standard, as described in section III-3, $\mathrm{d}$, page 172. A ground glass is placed in the plane of the photographic film and the image of a reference line is observed on this glass with an eyepiece while focusing. The reference line should be at the object distance intended for the interferometer while in the furnace. If the change in this object distance remains small, no further focusing will be necessary.

The adjustments required for a simple interferometer (that is, one having a single set of fringes) have been given in a previous publication [5]. A compound interferometer, such as that shown in figure 6 , 
imposes additional requirements on the adjustments, the most important of which is the requirement that the two or more sets of fringes shall be equally bright. The orientation of the interferometer image, relative to the exposing slit, is also more critical for the compound interferometer as it is necessary to have the images of all reference points fall in the exposing slit.

Plate $E$, figure 6 , is made wedge-shaped so as to eliminate the light reflected from its top surface. Consequently, the light that is transmitted by this plate, which forms the expansion fringes, does not return parallel to the light which forms the temperature fringes. That is, the two returning beams do not coincide and, consequently, separate images of the source are formed that are relatively displaced in the plane of aperture 19. As aperture 19 is equal, both in size and in shape, to either image of the source, and as these images do not coincide exactly, all the light from the interferometer cannot pass through this aperture.

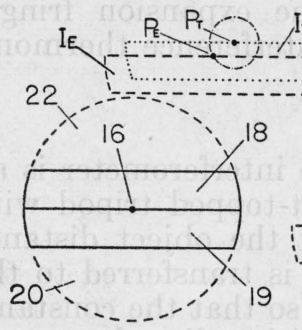

A

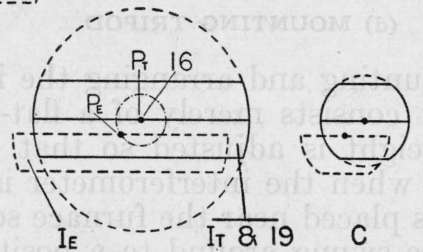

$\mathrm{B}$

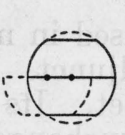

D

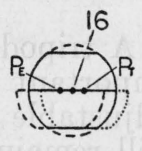

E

FIGURE 13.-Adjustments of light beams.

See text for_explanation.

The adjustments for securing maximum brightness of images, consistent with the requirements for equal brightness, are best explained with the aid of figure 13. The parts designated by numbers have already been defined. $I_{T}$ and $I_{E}$ are the images of the source, formed in the plane of aperture 19, by light of the thermometer and expansion fringes, respectively, whereas $P_{T}$ and $P_{E}$ represent the corresponding images of point 16 which is the intersection of the optic axis with this plane.

Figure $13, A$, represents the general condition produced when an interferometer, such as is shown in figure 6 , is placed on the mounting tripod, and the interferograph is swung into position for observing it. In general, neither of the two images, $I_{T}$ and $I_{E}$ (which are assumed to be equally bright), fall on aperture 19 and, consequently, the interference fringes cannot be seen by an observer looking into the eyepiece. An outline of the interferometer can be seen, however, by diffuse light from an outside source. This light serves to identify the proper location of the interferometer relative to the interferograph. By tilting the tripod the two images, $I_{T}$ and $I_{E}$, are caused to move relative to aperture 19 , whereas their positions relative to each other remain fixed. With diaphragm 17 (fig. 2) wide open and by tilting the mounting tripod, the refraction thermometer image, $I_{T}$, is easily brought into coincidence with aperture 19 , as is shown in figure $13, B$. 
This condition is attained when the temperature fringes appear brightest. The diaphragm opening is then reduced until the expansion fringes become quite faint. This condition indicates that only a small portion of the image, $I_{E}$, is coinciding with aperture 19 , as shown in figure $13, C$. Usually the brightness of the expansion fringes may be increased by rotating plate $E$ of figure 6 , without shifting it from its proper position, on the specimen, $S$. When plate $E$ is thus rotated, the point, $P_{E}$, moves on a circle with $P_{T}$ (which now coincides with 16) as its center. When $P_{E}$ is on the horizontal line through $P_{T}$, the expansion fringes are brighter than for any other angular rotation of the plate. This condition (represented by $13, D$ ) is reached when the total amount of light transmitted through aperture 19 becomes a maximum for this size of aperture; however, the two sets of fringes are still unequal in brightness. When the mounting tripod is further tilted in the proper direction, the condition shown in figure $13, E$, is produced, in which the intensity of the two beams becomes equal without changing the total amount of transmitted light.

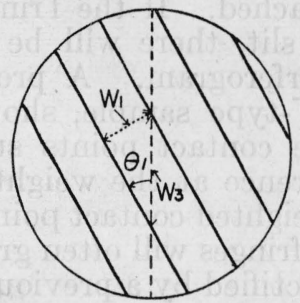

A

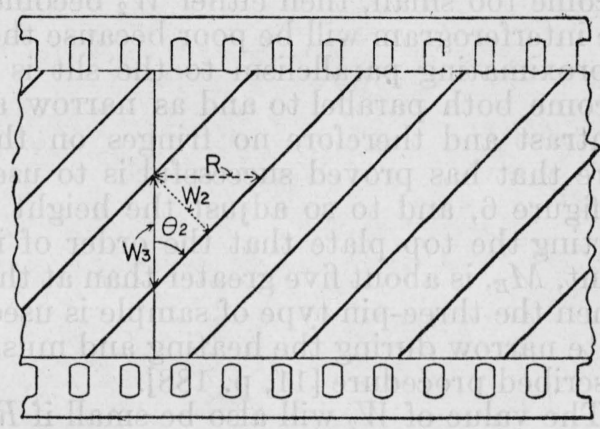

B

FiguRE 14.--Relation between interference fringes and interferograms.

$A$, Interference fringes; $B$, corresponding interferogram; $W_{1}$ and $W_{2}$, width of interferometer and interferogram fringes, respectively; $W_{3}$, expanse of fringe width along the slit; $\theta_{1}$ and $\theta_{2}$, angular orientation of interference and interferogram fringes, respectively; $R$, length of film passing a reference point during the passing of one fringe.

\section{ASSEMBLING THE INTERFEROMETER}

\section{PRELIMINARY CONSIDERATIONS}

For accuracy in reading the interferogram, the width of the bands (as in visual readings of the interferometer fringes) should be neither too wide nor too narrow. However, when flat interferometer plates are used, it sometimes happens that the tilting of the plates will cause the interferometer bands to become either much wider than the visible field of the interferometer or too narrow to be counted. These conditions occur when the angle formed by the two reflecting surfaces becomes either too small or too large. Such conditions make it impossible, when taking data visually, to read the fractional order of the fringes. However, this broadening or narrowing of the fringes is usually less troublesome with the interferograph, provided certain precautions are taken while assembling the interferometer. If the width $W_{1}$, (fig. 14) and orientation $\theta_{1}$ of the interferometer fringes 
are known, the corresponding width, $W_{2}$, and orientation, $\theta_{2}$ of the interferogram fringes are given by the equations,

$$
\begin{aligned}
W_{2} & =\left(R^{-2}+W_{3}^{-2}\right)^{-1 / 2} \\
\theta_{2} & =\tan ^{-1}\left(R W_{3}^{-1}\right)
\end{aligned}
$$

where $W_{3} \equiv W_{1} \csc \theta_{1}$ is the expanse of fringe width along the slit, and where $R \equiv V \dot{N}^{-1}$ is the length of film used per fringe. The quantities $V$ and $\dot{N}$ are the speed of the film motion and the rate of change in the order of interference, respectively.

An analysis of the above transformation equations show that $W_{2}$ can never exceed either $R$ or $W_{3}$. Therefore, if either of these quantities are prevented from becoming large, the width of the interferogram fringes cannot become too large for reading. If the surfaces of the interferometer plate are not flat (as is usually the case) it is quite obvious that the interferometer fringes can never become too wide.

The operator can never be sure that the fringes will not become too narrow during a test. If the width, $W_{1}$, of the interferometer fringes become too small, then either $W_{2}$ becomes too narrow or contrast on the interferogram will be poor because the condition of narrow fringes approximating parallelism to the slit is approached. If the fringes become both parallel to and as narrow as the slit, there will be no contrast and therefore no fringes on the interferogram. A procedure that has proved successful is to use the T-type sample, shown in figure 6 , and to so adjust the height of the contact points supporting the top plate that the order of interference at the weighted point, $M_{E}$, is about five greater than at the unweighted contact points. When the three-pin type of sample is used, the fringes will of ten grow quite narrow during the heating and must be rectified by a previously described procedure [11, p. 188].

The value of $W_{2}$ will also be small if $R$ becomes small. The value of $V$ is limited to finite values; that is, to $3 / 2,3$, and 11.4 inches of film per hour, for the instrument described above. Therefore, the only unavoidable condition that will cause $R$ to become too small is for $\dot{N}$ to become so large that the instrument is unable to provide a film speed sufficient to prevent excessive crowding of fringes. The largest value for $\dot{N}$ that permits satisfactory resolution of the fringes is that which corresponds to approximately 100 fringes per inch of film. To aid in preventing the fringes from becoming narrow, the initial value of $\theta_{1}$ should be small if the fringes are subject to rotation and changes in their width. In a stable fringe system, such as that of an interference thermometer-where the two reflecting surfaces are permanently bound-the value of $\theta_{1}$ may have any value that permits a reasonable value for $W_{3}$.

\section{ASSEMBLY PROCEDURE}

The preparation of the specimens which serve as spacers between the interferometer plates is fully described by Merritt [5], Saunders [11], and Johnson and Parsons [12].

The steps usually taken to assemble an interferometer similar to that shown in figure 6 are as follows:

With eyepiece 99 in position, the interference thermometer is placed on the mounting tripod so that an image of its reference line, $M_{T}$, will 
be perpendicular to the exposing slit. The beam of reflected light from the thermometer is adjusted for maximum brightness by adjusting the screws of the mounting tripod. The specimen, $S$, is placed in a position so that the exposing slit may be made to coincide simultaneously with the expansion and thermometer reference points, $M_{E}$ and $M_{T}$, respectively. The top plate, $E$, is then placed on the sample. At this stage the expansion fringes may or may not be visible, depending upon whether the image $\left(I_{E}\right.$, fig. 13$)$ falls in or without aperture 19 . In either case, by rotating plate $E$, the expansion fringes may be made almost as bright as the temperature fringes. The mounted interferometer is then carefully placed in the furnace and at approximately the same orientation relative to the base of the interferograph. Prism 1 is now brought into position, over the furnace, by rotating the interferograph about shaft 84 . The interferometer is releveled by means of the furnace leveling screws, 94 . A rotation of the furnace restores the relative angular orientation of the two reference points relative to the exposing slit. Eyepiece 99 is now replaced by the front part, 40 , of the camera box. A slight movement of the interferograph on table 97 while a magnifying glass ${ }^{7}$ is used to observe the interferometer through the exposing slit (opened to approximately $1 \mathrm{~mm}$ for this adjustment) will then bring the two reference points into coincidence with the exposing slit. Equal brightness of the two sets of fringes, together with maximum illumination of photographic film, is restored by a final adjustment of the furnace leveling screws. The interferometer is then properly mounted and ready for use.

\section{PHOTOGRAPHIC EXPOSURE}

Correct exposure for an interferogram requires good contrast and sufficient density for easy reading. The requirements for contrast are specified below but those for density must be ascertained by trial. These two factors depend both on the type of photographic film and the characteristics (light source and adjustments) of the interferograph.

\section{SELECTION OF PHOTOGRAPHIC FILM}

The type of photographic film to be used is an important feature in any photographic work. In the case of the interferograph, contrast and speed are the essential factors. The speed rating as specified by the manufacturers does not necessarily indicate the sensitivity of the film to any given spectral line. The relative speed factor for interferographic work with any line may nevertheless be determined from the specified speed rating, together with the spectral sensitivity curve of the film.

The speed of the film is important, as the more sensitive the film is to the light used, the narrower the slit may be made, and the faster the film may be moved past the slit. A narrow slit gives high resolution which is very important when the interferometer bands are narrow and nearly parallel to the slit, and also when they are moving rapidly. The ability to record fast-moving fringes is necessary when-

\footnotetext{
7 This magnifying glass may be a simple lens of wide aperture, and it may be permanently attached to the inside wall of the camera box. It should be attached to the fixed part (40, fig. 8$)$ and well back of the sprocket so that it does not interfere with the removal of part 39 when opening and closing the camera.
} 
ever expansion tests are made at high heating rates, whenever moderate rates of heating are used with materials that have a high expansivity, and when following the changes in density of crystals during the transformation from one form to another. When yellow light from the helium source is used, any of the fast panchromatic films are satisfactory.

In deciding upon the required spectral sensitivity distribution of the film, the available light source must be considered. If a helium source is used, it is desirable to select panchromatic film for use with the yellow line because this film is highly sensitive to yellow light. This combination gives plenty of speed and also produces good visibility for visual adjustments. However, greater contrast can be obtained by using some of the noncolor-sensitized films. If the latter type of film is used, blue light is required because such films are insensitive to greens, yellows, and reds but are highly sensitive to the blues. Consequently, high contrast may be obtained by using the blue line $(\lambda=4471 \mathrm{~A})$ of helium with a noncolor-sensitized film. The blue line of helium is only about one-half as intense as the yellow line, and the product of sensitivity of film by intensity of the line may be less than the corresponding product when the yellow line with panchromatic film is used. The combination of yellow light with panchromatic film is recommended for temperatures up to $675^{\circ} \mathrm{C}$ and blue light with noncolor-sensitized films for tests that require higher temperatures.

\section{CONTRAST}

If the area of the effective source is large, the cosine error in the interference phenomena is large and the fringes appear washed out from lack of contrast. The smaller the source the greater will be the contrast, but a compromise must be considered between contrast and amount of light needed for the record. Contrast in the interferogram depends also upon the width of the exposing slit. That is, the interferograph will reproduce the contrast visually observed in the interference phenomena only if the change in the order of interference is small during the exposure of any point on the film. The best results require the use of a source with the largest area that will give reasonable contrast in the interference fringes, the use of the widest exposing slit that will give reasonable reproduction of contrast, and the making of other necessary variations in exposure by changing the intensity of the source and by varying the film speed.

\section{PHOTOGRAPHIC DENSITY OF FILM}

Assuming that a reasonable amount of contrast is attained in the interference phenomena, the best exposure is that for which the photographic density approaches zero at the center of the light fringes. The only remaining factors available for controlling exposure (after the consideration already given to contrast) are the intensity of the light source and speed of film motion. The speed of the film motion is determined mainly by the rate of change in the order of interference. Consequently, the intensity of the source is the final factor in deciding the photographic density to be obtained. Hence, if the film speed is changed during a test, the intensity of the source must be changed accordingly to compensate for the resultant change in exposure time. 


\section{OTHER APPLICATIONS OF THE INTERFEROGRAPH}

The interferograph is especially applicable to the measurement of thermal expansions. However, it is equally well adapted to the measurement of many other interference phenomena. It is suggested that this apparatus furnishes a convenient means of obtaining data for those investigations that require a determination of the absolute order of interference, as by the method of coincidence; for the recording of changes in the order of interference that are very large, such as are encountered in the measurement of the standard meter bar; for the recording of changes in interference fringes over long periods of time, as in ether-drift experiments; and for other investigations that present difficulties to visual observation.

A simple arrangement for measuring the rate of disappearance of strain in a plate of optical glass, such as $E$ of figure 6 , is to replace sample $S$ by a nonreflecting sheet of material or by removing the sample $S$, fine grinding the top part of plate $T$ under plate $E$ and then laying plate $E$ directly on plate $T$. The arrangement is completed by placing a nicol to the left of and close to diaphragm 17 of figure 2, so that all light passing from aperture 18 to lens 15 and from lens 15 to aperture 19 will pass through the nicol. The light after passing through the nicol will be plane polarized. It traverses the strained plate, $E$, is reflected (in part) at the lower surface of this plate and again traverses the plate from which it returns through the nicol prism to aperture 19 and on to the photographic film. The nicol prism serves both as a polarizer and an analyzer. The resultant interferogram will contain a measure of time, temperature, and changes in double refraction effects. A polaroid screen, placed anywhere between lens 15 and diaphragm 17, serves as well as a nicol. Greater accuracy may be attained by having the specimen shaped so as to cause the light to suffer several internal reflections before returning to the analyzer.

Preliminary experiments indicate the applicability of the interferograph to the determination of visibility curves of spectrum lines [13] that are composed of doublets, triplets, or single bright lines with associated satellites. The intensity measurements for the various orders of interference, on the interferograms, are easily and rapidly made with a suitable photoelectric densitometer.

\section{RESULTS}

To illustrate an application of the interferograph to expansion work, two interferograms of the expansion of quartz in the range between room temperature and $650^{\circ} \mathrm{C}$ are reproduced. The interferogram (shown near the bottom of fig. 16, A) was produced while the sample was being heated at a constant heating rate of 2.4 degrees per minute and while 3 inches of film per hour was used. Under these conditions it is impossible to follow the fringe count in the neighborhood of the transformation temperature, $573.3^{\circ} \mathrm{C}$. $[14]^{8}$. Consequently, in order to record this part of the data legibly, a second interferogram (a part of which is enlarged and shown in fig. 16, B) was produced which differs from the first only in that the heating rate was lower and the film speed

${ }^{8}$ Bates and Phelps have found that the start of the inversion of $\alpha$-quartz to $\beta$-quartz occurs at $573.3^{\circ} \mathrm{C}$. This temperature is accepted as one of the base pointsfor calibrating the interference thermometer. 
greater. Thus, when $573^{\circ} \mathrm{C}$ was reached, the heating rate was approximately 1.6 degrees per hour and the speed of the film was 11.4 inches per hour. These conditions allot 7 inches of film to each degree rise in temperature, whereas in the interferogram of figure $16, \AA$, approximately one-fiftieth of an inch per degree was allowed. This combination of changes in film speed and heating rate is equivalent to a magnification of approximately 330 in the longitudinal direction of the film and was sufficient to resolve the fringes even while the samples was passing through the transformation from low to high quartz.

A U-shaped sample was used as this shape permits an image of one of the unweighted contact points ( $M_{2}$ of fig. 16) to be included, along: with the weighted contact point, $M_{1}$, in the exposing slit. Thus, including that of the thermometer, there are three reference lines in each of the interferograms. The two end-contact points of the $U$-shaped sample were alined with the thermometer reference point so that the images of all three could be included in the exposing slit. As usual, one of the contact points of the sample was loaded by shifting the center of the top interferometer plate toward it.

The purpose of including a second reference point in the field of the expansion fringes is to enable the interpreter to follow the fringe count through the transformation at $573.3^{\circ} \mathrm{C}$. A small and usually unavoidable temperature gradient between the two parts of the sample that are imaged in the exposing slit causes them to transform at different times. Consequently, the fringes may be moving quite slowly at one of these reference points while they are moving rapidly at the other where the transformation is occurring.

The initial length ${ }^{9}$ of the sample was obtained from the approximate length (measured with micrometer calipers) by applying the "theory of coincidence" to the fractional orders of interference produced by each of several known light sources. The application of this principle has been described by Meggers [15] and Perot and Fabrey [9]. A photograph of the interference fringes, produced by each of the several sources, is obtained by taking a series of still pictures with the exposing slit wide open. To prevent double exposure the film is moved forward after each exposure by an amount slightly greater than the width of the slit. The lines ${ }^{10} 4358,5461,5770,5791 \mathrm{~A}$ of mercury and $4471,4713,4922,5016$, and 5876 of helium were used for this record.

After the length of the sample at room temperature is known, the absolute order of interference, corresponding to any of the fringes crossing the exposing slit, is also known. This enables the interpreter to label the interferogram fringes (at the starting end of the interferogram) with numbers that correspond to the absolute order of interference of the interferometer fringes producing these fringes. All other interferogram fringes can then be labeled by counting from the original order. These numbers are written above the interferogram in figure $16, \mathrm{~A}$ and the last two significant figures of these numbers are written on the corresponding fringes of figure $16, \mathrm{~B}$. Obviously the count of the fringes in figure $16, \mathrm{~A}$, cannot be followed through the transformation temperature but it can be followed in figure 16, B. The order of the fringes above the transformation temperature, in figure $16, \mathrm{~A}$, is easily ascertained from figure $16, \mathrm{~B}$, as the order of the

$\checkmark$ A micrometric determination of the specimen length is sufficient for most expansion work.

${ }_{10}$ Usually five well-known lines are sufficient. 


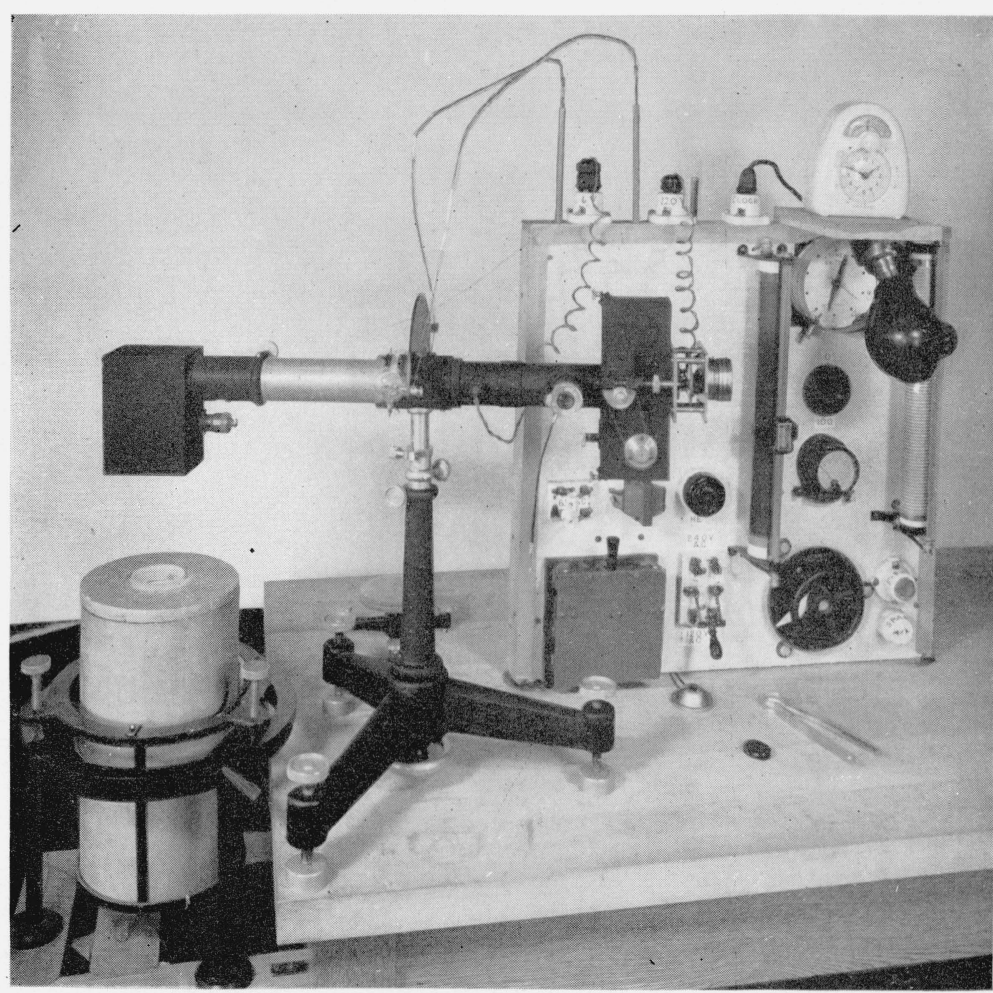

FIGURE 15.-Assembly of the thermal expansion apparatus. 
TIME, 42 MINUTES PER DIVISION

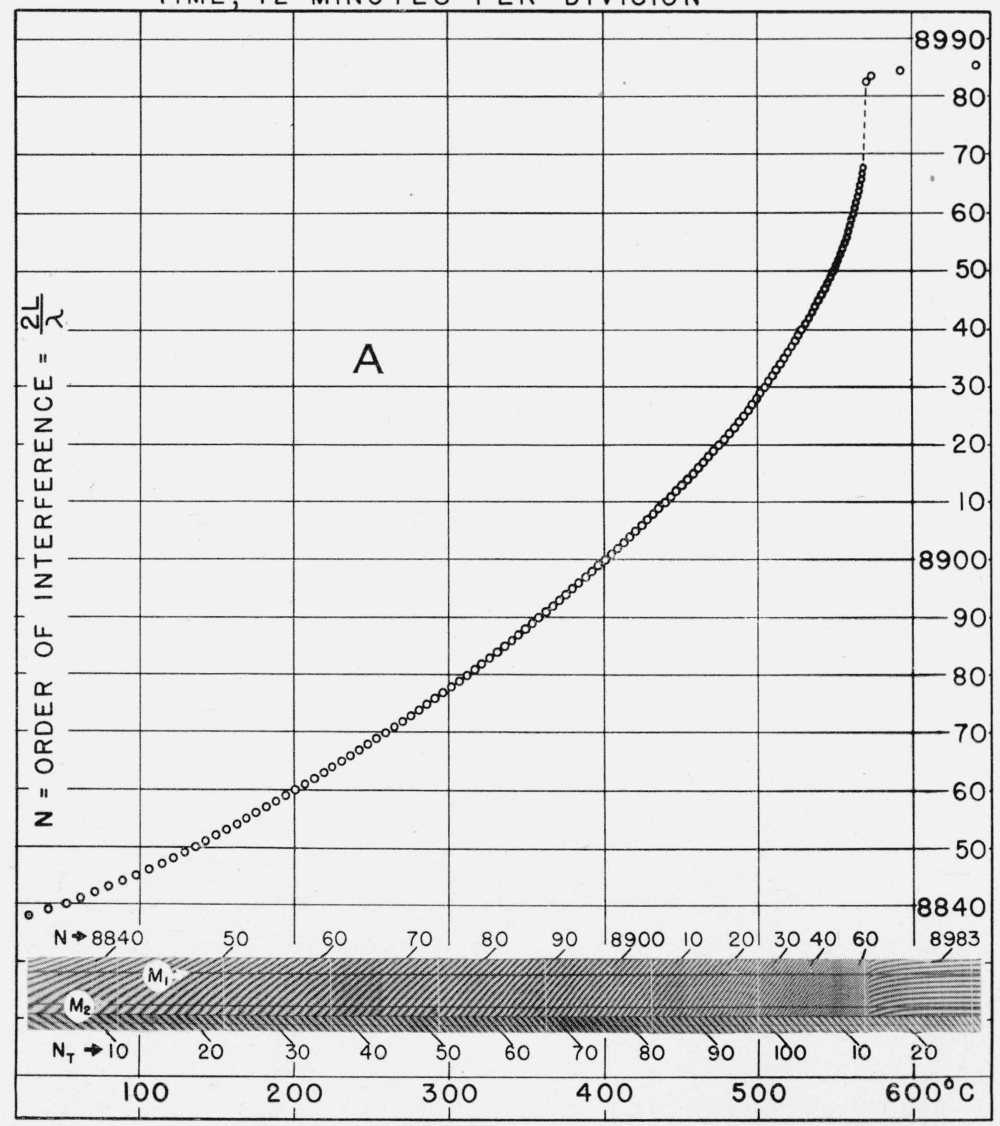

TEMPERATURE

FIGURE 16, A.-Interferograms of the thermal expansion of quartz with graphical representations of the included data

$N$, Absolute order of interference that produced the corresponding expansion fringes; $N_{T}$, arbitrarily chosen numbers assigned to temperature fringes for identification and calibration purposes; $L$, length of sample; $\lambda$, wavelength of light used for producing interference; $M_{1}$, reference line correspondirg to weighted reference point; $M_{2}$, reference line corresponding to unweighted reference point. The curve of $A$ and curve 1 of $B$ were obtained from the weighted reference line. Curve 2 of $B$ was obtained from the unweighted reference line. The numbers appearing on the interferogram of $B$ are the last two significant figures of $N$. The interferograms of $A$ and $B$ were made while approximately 0.02 and 7.0 inches of film wasused' respectively, for each degree rise in temperature. 
TIME, APPROXIMATELY 3.7 MINUTES PER DIVISION

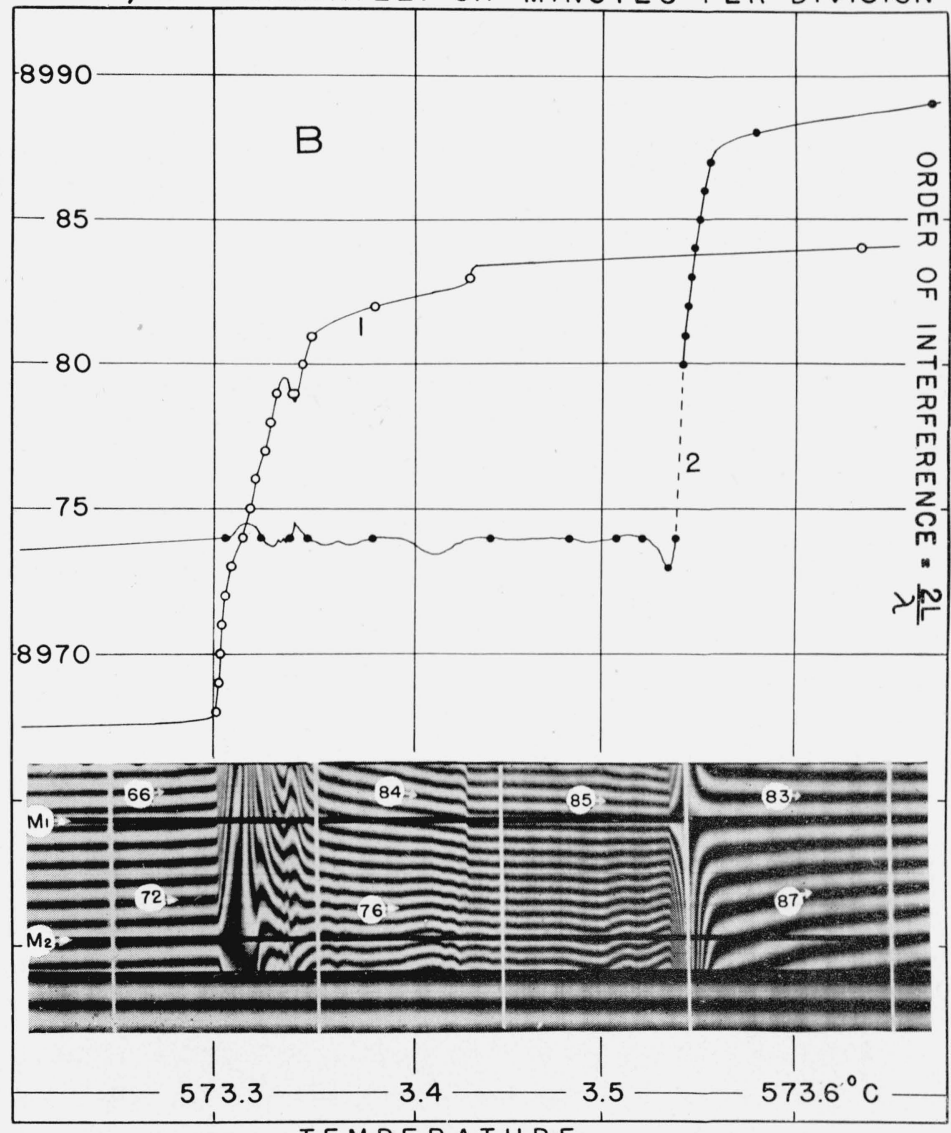

TEMPER ATURE

Figure 16, B.

(See fig. 16, A, for explanation.) 

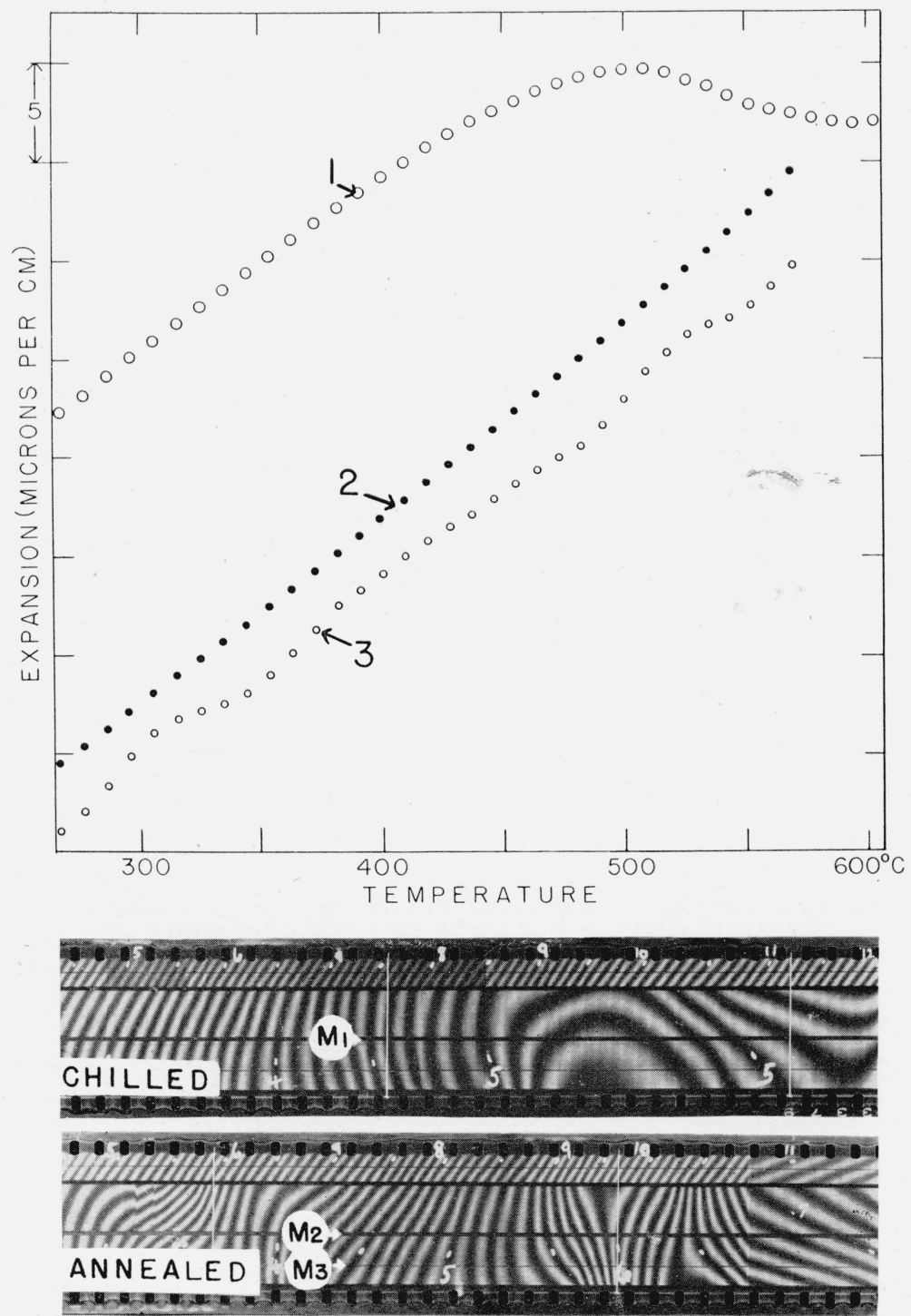

FiguRE 17.-Interferograms and corresponding expansion curves for a giass in both the chilled and annealed states.

Curves 1 and 2 were computed from data that were taken along the two parts, $M_{1}$ and $M_{2}$, of the weighted reference line, whereas curve 3 was computed from the un weighted referenceline $M_{3}$. The relative position of the curves along the axis of abscissas were arbitrarily chosen to prevent congestion and to sa ve space. 
expansion fringes in the two interferograms will agree at all temperatures of this range.

Plots of the absolute orders of interference versus temperature (and time) are given above the interferograms in figure 16 . The ordinates represent the absolute length of the specimen in terms of half wavelengths of the light used. The abscissas represent both temperature and time; the time scale is at the top and the temperature scale is at the bottom of the graphs. This simultaneous representation of both temperature and time by the abscissas of a graph is possible because the heating rates were approximately constant. The length of the film exposed was used as a measure of the elapsed time, and the temperatures were determined from the calibration sheet of the interference thermometer. No other computation was necessary in preparing the plotted points, as a drawing board and T-square served to transform the data from the interferogram directly to the graph being plotted.

It will be noted that the slopes ( $\theta$, fig. 14$)$ of the expansion fringes on the interferogram and the corresponding parts of the expansion curves, plotted in such a manner, should be closely related if the heating rate and film speed are constant, if there is no tilting of the two reflecting surfaces relative to each other, and if the wavelength is kept constant by performing the test in vacuum. The correlation between the interferograph fringes and the corresponding expansion curves is quite apparant in figure 16 although some tilting and variation in the heating rate, as well as gradients, were present.

Discontinuities such as those in the interferograms of figure 16 are caused by a rapid change in the expanse of fringe width ( $W_{3}$, fig. 14) along the exposing slit. That is, this expanse increases from a normal value to infinity and then returns to normal after reversing the direction of the fringe count along the slit. This change in the expanse of fringe width along the slit is caused by a tilting of the top interferometer plate relative to the bottom plate. ${ }^{11}$ The observed effect (when looking into the eyepiece) may be either a change in width without rotation, a rotation without a change in width, or both a rotation and change in width, the latter being the usual case. The photographed effects resulting from a rotation of fringes are usually not distinguishable from those resulting from a change in the width of the fringes.

If the tilting of the interferometer plate proceeds slowly, as is often the case, the photographed effects include a progressive change in the slope of the interferogram fringes. If the tilt occurs suddenly, which frequently occurs, a discontinuity is produced. Both of these effects are shown in a previously published [3] interferogram. A drawing that represents a part of this interferogram is shown in figure 18. This drawing is included here as an aid in explaining the interpretation of results in the neighborhood of such discontinuities. The numbers occurring in this drawing are inserted to indicate the fringe count from room temperature. The previously published results

\footnotetext{
11 In the case of the first discontinuity of figure 16, B, the tilt of the top interferometer plate was caused by a rapid expansion of the weighted part of the spacer that produced $M_{1}$. As this part of the spacer increased in length and became equal to that part of the spacer corresponding to $M_{2}$ the dark interference fringe, No. 74 , which produced the interferogram fringe then coinciding with reference line $M_{2}$, became parallel to the slit and this fringe then covered both reference points. After this occurred the movement of the interference fringes, in the slit, was reversed as the numbering of the interferogram fringes indicate. At the second pronounced discontinuity, the 84th fringe was on the weighted reference point $M_{1}$ when the unweighted spacer passed through the transformation as it expanded very rapidly. This effect again reversed the direction of motion of the interference fringes along the slit.
} 
were taken from the weighted reference line, $M_{1}$. Reference line $M_{2}$ was produced by a reference point at the median of the triangle formed by the three supporting points of the top interferometer plate. When the first discontinuity, shown near the center of figure 18, occurred fringe 40 was on the weighted reference point. A sudden tilting of the plate caused a corresponding change in the expanse of fringe width along the exposing slit. It has already been demonstrated [11] that this rotation of the fringes always occurs about an axis that passes through the weighted contact point. Therefore fringe 40 still passed through the weighted reference point after the sudden tilt occurred. It is known that the sample was expanding uniformly from a consideration of other interferograms, which recorded the changes in this material through this same temperature range. Consequently, the next fringe to cross the weighted reference line is known to have been 41 . The discontinuity shown near the right end

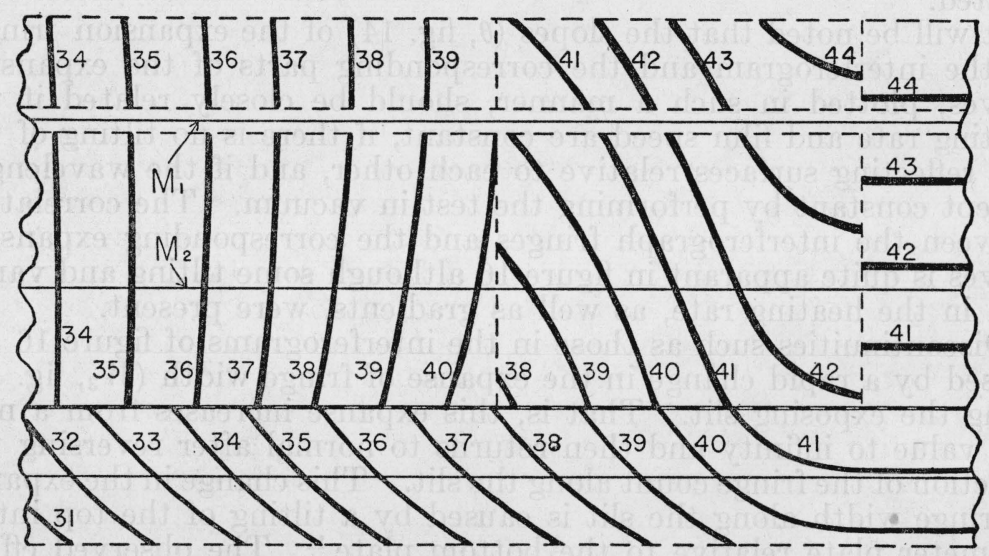

FIgURE 18.-Discontinuities in interferograms.

The inserted numbers represent the orders of interference, counted from room temperature; $M_{1}$ and $M_{2}$ are the weighted and unweighted reference lines, respectively. The upper and lower sets of fringes are expansion and temperature fringes, respectively.

of figure 18 occurred when the temperature of the sample was constant and when the fringe count at the weighted reference point was 43.7 , and it is again apparent that no change in the fringe count occurred at the weighted reference point. If the expansion data had been obtained from a fringe count taken at the median reference line, $M_{2}$, the magnitude of the sudden change in the fringe order at this point would not have been noted and the results corresponding to points above this temperature would have been in error to the extent of one fringe at least. The correct fringe count can always be followed by accepting the fact that no sudden change occurs in the order of interference at a properly weighted reference point.

For representing expansion data graphically, the usual procedure is to tabulate the data obtained from points along the selected reference lines of the interferograms and from these data to compute the temperatures and corresponding changes in length. The results are then plotted without further reference to the interferograms. Such a graphical representation, along with the interferograms from which the original data were taken, is introduced in figure 17 to show the 
close correlation between an interferogram and the corresponding computed curve, even when it passes through maximum and minimum points. The location of such points are more readily obtained from the interferogram than from the curves. The same is also true of critical points in the expansion curves of crystals (fig. 16). The interferograms in figure 17 are parts of the record of a glass that was previously chilled from its molten state. The chilled sample was heated at a constant rate of 3 degrees centigrade per minute to 610 degrees centrigrade and annealed at this temperature. It was then cooled to room temperature and, without dismantling the interferometer, a second expansion test was made on the sample, now in an annealed state. The complete record is not shown here as the excluded parts contained nothing of current interest. Curves 1 and 2, which are computed from data taken along reference lines $M_{1}$ and $M_{2}$, are typical expansion curves for chilled and annealed glass, respectively [16]. In this figure, reference lines $M_{1}$ and $M_{2}$ are produced by the weighted reference point, whereas $M_{3}$ is typical of accidental marks formed by particles of dust that get into the slit during a test. Curve 3 was computed from data obtained at $M_{3}$ and is included to demonstrate the effect of errors when the data are not taken along the weighted reference point. The lower interferogram also shows the effect resulting from the squirming of the interferometer fringes that is caused by changes in the thickness of the air films between the specimen and interferometer plates at the unweighted reference points. It is these air-film effects that produce the irregularities that are obvious errors in the data from which curve 3 was computed. The effect of a change in the film speed, while heating the interferometer at a constant rate, is shown near the right-hand end of this interferogram. The upper set of fringes in each interferogram are the temperature fringes. The white numbers inserted in the interferograms were written on the negatives to aid in the fringe count when the data were translated from the interferograms to numerical values.

\section{PROPOSED REFINEMENTS}

As a result of experience gained with the interferograph, a few changes in its design are proposed. Although not affecting the performance, these changes incorporate certain desirable features. For example, the constant-deviation prism described above is quite massive and although no difficulty has been experienced, there is a possibility of injury to it due to heat shocks when measuring expansions to very high temperatures. To reduce this danger a new prism has been designed in which all dimensions except thickness have been reduced to one-half, thus reducing the mass to one-fourth that of the original prism. This reduction in the size of the prism does not reduce the dimensions of the aperture in the directive parallel to the exposing slit. The visible field is limited by the collimating lens and the two horizontal edges of the entrance face of the new prism. Thus the visual field has the general shape of the fringe field shown in figure $1, C$, with the two straight sides separated by an amount equal to the radius of the collimating lens.

It is desired to call attention to the difficulty sometimes experienced in transferring the interferometer from the mounting tripod to the furnace. This is minimized by first transferring the interferometer from the tripod to a small cup fitted with a bail-like handle, and then 
lowering it into the furnace by means of a hook. Even so, the operation frequently involves several unsuccessful trials. Therefore, in place of this procedure it is proposed to modify the furnace so that the mounting of the interferometer may be done directly in the position it will occupy during the observations. This may be accomplished in a number of ways. The furnace may be constructed so that onehalf may be removed while the interferometer is being set up, and then replaced. Or the interferometer may be supported on a pillar independent of the furnace and the furnace elevated and swung out of line while adjustments are made.

\section{$\mathrm{X}$. CONCLUSIONS}

The experimental instrument described herein was found to perform excellently. The results from a large number of tests have conclusively demonstrated the adequacy of its mechanical performance. An outstanding advantage of the interferograph over visual instruments is that the recorded interferograms are permanent records that are available for repeated critical reviews of their interpretation. The small amount of attention required for the operation of this instrument allows the operator to prepare new specimens for later tests and to compile the data from previous tests during the photographic recording.

\section{REFERENCES}

[1] F. C. Nix and D. MacNair, Interferometric-dilatometer with photographic recording, Rev. Sci. Instr. 12, 66 (1941).

[2] A. Trowbridge, Photography of moving interference fringes, J. Opt. Soc. Am. 6, 195 (1922).

[3] J. B. Saunders, Interferometer measurements on the expansion of iron, J. Research NBS 33, 75 (1944) RP1597.

[4] C. G. Peters and C. H. Cragoe, Measurements on the thermal dilation of glass at high temperatures, Sci. Pap. BS 16, 457 (1920) S393.

[5] G. E. Merritt, The interference method of measuring thermal expansion, BS J. Research 10, 59 (1933) RP515.

[6] Ph. Pellin and A. Broca, Spectroscope à déviation fixe, J. phys. 8, 314 (1899).

[7] A. Pérard, Quelques études particulières au dilatomèter Fizeau, Travaux et Mémoires du Bureau International des Poids et Mesures, XIX, 21 (1932).

[8] C. H. Cartwright, Treatment of camera lenses with low reflecting films, J. Opt. Soc. Am. 30, 110 (1940).

[9] A. Perot and Ch. Fabry, Méthodes interférentielles pour la mesure des grandes épaisseurs et la comparison des longuers d'onde, Ann. Chim. phys. 16, 289 (1899).

[10] M. Luckiesh, L. L. Holladay, and R. H. Sinden, An interference thermometer and dilatometer combined, J. Franklin Inst. 194, 251 (1922).

[11] J. B. Saunders, Improved interferometric procedure with application to expansion measurements, J. Research NBS 23, 179 (1939) RP1227.

[12] W. H. Johnson and W. H. Parsons, Thermal expansion of concrete aggregate materials, J. Research NBS 32, 101 (1944) RP1578.

[13] A. A. Michelson, On the application of interference methods to spectroscopic measurements, Phil. Mag. [5] 34, 280 (1892).

[14] F. J. Bates and F. P. Phelps, A suggested new base point on the thermometric scale and the $\alpha \rightleftarrows \beta$ inversion of quartz, BS Sci. Pap. 2\%, 315 (1927) S557.

[15] W. F. Meggers, Notes on comparisons of lengths of light waves by interference methods, and some wave lengths in the spectrum of Neon gas, Bul. BS 12, 203 (1915) S251.

[16] A. Q. Tool, D. B. Lloyd, and G. E. Merritt, Dimensional changes caused in glass by heating cycles, BS J. Research 5, 642 (1930) RP219.

[17] M. A. Arnulf, Un appareil enregistreur pour la mesure des dilations des verres, Rev. optique 3, 270 (1924).

[18] R. H. Sinden, An interferential dilatometer employing automatic photography, J. Opt. Soc. Am. 15, 171 (1927).

Washington, May 10, 1945. 